Pacific

Journal of

Mathematics

A DESCRIPTION OF CERTAIN AFFINE OPEN SUBSCHEMES THAT FORM AN OPEN COVERING OF $\mathbf{H} i l b_{\mathbf{A}_{k}^{2}}^{n}$

Mark E. Huibregtse

Volume $204 \quad$ No. 1

May 2002 


\title{
A DESCRIPTION OF CERTAIN AFFINE OPEN SUBSCHEMES THAT FORM AN OPEN COVERING OF $\mathrm{Hilb}_{\mathrm{A}_{\mathrm{k}}^{2}}^{n}$
}

\author{
Mark E. Huibregtse
}

\begin{abstract}
In this paper, we study certain affine open subschemes of the Hilbert scheme of $\boldsymbol{n}$ points of the affine plane. We express the coordinate rings of these subschemes explicitly as quotients of polynomial rings; as an application, we give sufficient conditions for these subschemes to be isomorphic to $2 n$-dimensional affine space.
\end{abstract}

\section{Introduction.}

Let $\mathrm{k}$ be an algebraically closed field of any characteristic, $\mathrm{A}_{\mathrm{k}}^{2}=\operatorname{Spec}(\mathrm{k}[x, y])$ the affine plane over $\mathrm{k}$, and $\mathrm{Hilb}_{\mathrm{A}_{\mathrm{k}}^{2}}^{n}=\mathbf{H}^{n}$ the Hilbert scheme parameterizing 0 -dimensional closed subschemes

$$
\operatorname{Spec}(\mathrm{k}[x, y] / I) \subseteq \mathrm{A}_{\mathrm{k}}^{2}
$$

having length $n$, that is,

$$
\operatorname{dim}_{\mathrm{k}}(\mathrm{k}[x, y] / I)=\text { colength of } I=n .
$$

In particular, the k-points of $\mathbf{H}^{n}$ are in natural bijective correspondence with the ideals $I \subseteq \mathrm{k}[x, y]$ of colength $n$; we often identify the ideal $I$ with its associated point $I \in \mathbf{H}^{n}$. The correspondence is defined by the universal closed subscheme $Z_{n} \subseteq \mathbf{H}^{n} \times \mathrm{A}_{\mathrm{k}}^{2}$, which is finite and flat of degree $n$ over $\mathbf{H}^{n}$ via the first projection: The k-point $t \in \mathbf{H}^{n}$, given as a map

$$
t: \operatorname{Spec}(\mathrm{k}) \rightarrow \mathbf{H}^{n},
$$

corresponds to the closed subscheme

$$
Z_{t}=\operatorname{Spec}(\mathrm{k}) \times_{\mathbf{H}^{n}} Z_{n} \subseteq \operatorname{Spec}(\mathrm{k}) \times_{\mathbf{H}^{n}}\left(\mathbf{H}^{n} \times \mathrm{A}_{\mathrm{k}}^{2}\right) \approx \mathrm{A}_{\mathrm{k}}^{2} .
$$

In a recent paper [7], Haiman defines, for each partition

$$
\mu=\left(p_{1}, p_{2}, \ldots, p_{\ell}\right) \text { of } n, \text { with } p_{1} \geq p_{2} \geq \cdots \geq p_{\ell}>0,
$$

an open affine subscheme $U_{\mu} \subseteq \mathbf{H}^{n}$, as follows: We first encode the Ferrers' diagram of $\mu$ as an arrangement of monomials in $x$ and $y$, with the parts 
corresponding to the rows. For example, if $\mu=(4,3,1)$, the diagram is

$$
\begin{array}{cccc}
x^{2} & & & \\
x & x y & x y^{2} & \\
1 & y & y^{2} & y^{3}
\end{array}
$$

we call the monomials in the diagram the partition monomials, and we write $(h, k) \in \mu$ to denote that $x^{h} y^{k}$ is a partition monomial. (The display can be extended in the obvious way to comprise all monomials in $x$ and $y$; when this is done, the monomial $x^{r} y^{s}$ resides in row $r$ and column $s$.) We then define $U_{\mu}$ (as a point set) to be

$$
\begin{aligned}
U_{\mu} & =\left\{I \in \mathbf{H}^{n} \mid \mathrm{k}[x, y] / I \text { is spanned by the partition monomials }\right\} \\
& =\left\{I \in \mathbf{H}^{n} \mid \text { the partition monomials are a } \mathrm{k} \text {-basis of } \mathrm{k}[x, y] / I\right\} .
\end{aligned}
$$

Haiman proves that $U_{\mu}$ is an open affine subscheme of $\mathbf{H}^{n}$, and describes an infinite family of regular functions on $U_{\mu}$ that generates the coordinate ring $\mathcal{O}_{U_{\mu}}[7$, Proposition 2.1]. These functions arise as follows: For any $I \in U_{\mu}$, and monomial $x^{r} y^{s}$, we have a unique expansion

$$
x^{r} y^{s} \equiv \sum_{(h, k) \in \mu} c_{h k}^{r s}(I) x^{h} y^{k} \quad(\bmod I) ;
$$

as $I$ varies over $U_{\mu}$, the coefficients in this expansion define functions $c_{h k}^{r s}$ on $U_{\mu}$. Haiman shows that the set

$$
\left\{c_{h k}^{r s} \mid(h, k) \in \mu, \text { and all }(r, s)\right\}
$$

generates $\mathcal{O}_{U_{\mu}}$ as a k-algebra; he also identifies a $2 n$-member subset of the generating functions that is a set of local parameters at the point

$$
I_{\mu}=\left(\left\{x^{r} y^{s} \mid(r, s) \notin \mu\right\}\right) \in U_{\mu},
$$

a monomial ideal that is the "origin" of $U_{\mu}$ in the sense that all the (nontrivial) functions $c_{h k}^{r s}$ vanish there. Haiman gives a new proof of the well-known fact that $\mathbf{H}^{n}$ is nonsingular (and irreducible) by reducing the question to the nonsingularity of $\mathbf{H}^{n}$ at monomial ideals (each of which is $I_{\mu}$ for some $\mu$ ), for which his explicit local parameters provide an affirmative result [7, Proposition 2.4, Corollary 2.5].

The purpose of this paper is to give a fuller description of $U_{\mu}$ and of the restriction

$$
Z_{U_{\mu}}=\operatorname{Spec}\left(\mathcal{O}_{U_{\mu}}[x, y] / \mathcal{J}_{\mu}\right)
$$

of the universal closed subscheme $Z_{n}$ to $U_{\mu}$. We begin by identifying a finite subset $\mathfrak{c}_{\mu}$ of the functions $c_{h k}^{r s}$ that generates the k-algebra $\mathcal{O}_{U_{\mu}}$. More precisely, Proposition 3.1.2 states that the set

$$
\mathfrak{c}_{\mu}=\left\{c_{h k}^{r s} \mid x^{r} y^{s} \text { is a leading monomial and }(h, k) \in \mu\right\}
$$


generates $\mathcal{O}_{U_{\mu}}$, where a leading monomial is a monomial that lies either immediately to the right of a row or immediately above a column in the diagram of $\mu$ (see Figure 1). We then prove in Proposition 3.2.1 that the

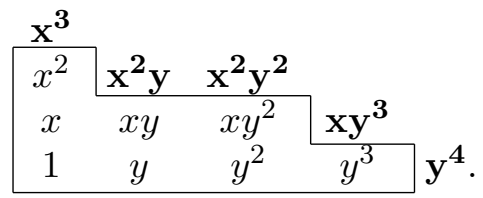

Figure 1. The diagram of $\mu=(4,3,1)$ with the partition monomials enclosed in a box and the leading monomials shown in boldface.

polynomials

$$
g_{r s}=x^{r} y^{s}-\sum_{(h, k) \in \mu} c_{h k}^{r s} x^{h} y^{k} \in \mathcal{O}_{U_{\mu}}[x, y]
$$

corresponding to the leading monomials $x^{r} y^{s}$ generate the ideal $\mathfrak{I}_{\mu}$ cutting out the universal closed subscheme over $U_{\mu}$. A simple algorithm (Algorithm 4.2.1) yields a basis of the first syzygy module of the generators $g_{r s}$ (Corollary 4.4.1), and hence a free resolution of the $\mathcal{O}_{U_{\mu}}[x, y]$-algebra $\mathcal{O}_{U_{\mu}}[x, y] / \mathcal{J}_{\mu}$ (Corollary 4.4.2); moreover, the $g_{r s}$ can be recovered (up to sign) as the maximal minors of the matrix whose rows are the members of the syzygy basis (Theorem 4.4.4).

To express the ring $\mathcal{O}_{U_{\mu}}$ concretely as a quotient, we introduce the set of indeterminates

$$
\mathfrak{C}_{\mu}=\left\{C_{h k}^{r s} \mid c_{h k}^{r s} \in \mathfrak{c}_{\mu}\right\},
$$

and define the surjection

$$
u_{\mu}^{*}: \mathrm{k}\left[\mathfrak{C}_{\mu}\right] \rightarrow \mathcal{O}_{U_{\mu}}, C_{h k}^{r s} \mapsto c_{h k}^{r s},
$$

which is the comorphism of a closed immersion

$$
u_{\mu}: U_{\mu} \rightarrow \operatorname{Spec}\left(\mathrm{k}\left[\mathfrak{C}_{\mu}\right]\right) .
$$

We give two different (computable) sets of generators for the kernel $\mathfrak{R}_{\mu}$ of the map $u_{\mu}^{*}$ (Theorem 5.1.1 and Theorem 6.1.1); the first of these is obtained as a byproduct of the (generalized) syzygy algorithm, and the second emerges from the recovery of the $g_{r s}$ as subdeterminants of the syzygy matrix. As an application, we obtain sufficient conditions on $\mu$ for $U_{\mu}$ to be an affine cell in $\mathbf{H}^{n}$, that is, an open subscheme isomorphic to $\mathrm{A}_{\mathrm{k}}^{2 n}$ (Corollary 7.3.2 and Corollary 7.5.1); these conditions subsume some important special cases, 
including $\mu=(1,1, \ldots, 1)$, which is discussed by Haiman [7, Corollary 2.8], and $\mu=(r, r-1, r-2, \ldots, 1)$, for which

$$
I_{\mu}=\left(x^{r}, x^{r-1} y, \ldots, y^{r}\right)=(x, y)^{r}
$$

is a "fat point" ideal (see Remarks 7.3.3).

We end the introduction with the following brief table of contents that summarizes the organization and contents of the paper.

Section 1: Introduction.

Section 2: Hilb $\mathrm{A}_{\mathrm{k}}^{2}$ and the affine open subschemes $U_{\mu}$. Summarizes the definitions and results from Haiman's paper [7] needed in the sequel, including certain relations among the functions $c_{h k}^{r s}$, and Haiman's local parameters at the point $I_{\mu}$. We also show in Section 2.5 that Haiman's result can be used to obtain local parameters at each point of $\mathbf{H}^{n}$.

Section 3: A finite set of generators of $\mathcal{O}_{U_{\mu}}$. Obtains the finite generating set $\mathfrak{c}_{\mu}$ of $\mathcal{O}_{U_{\mu}}$, and proves that the polynomials $g_{r s}$ (1) generate the ideal $\mathfrak{I}_{\mu}$.

Section 4: A free resolution of $\mathcal{O}_{U_{\mu}}[x, y] / \mathfrak{I}_{\mu}$. Presents the algorithm for computing a basis of the first syzygy module of the polynomials $g_{r s}$. (To prepare for subsequent sections, we study this algorithm in greater generality than is needed for the immediate application.) Consequences of the syzygy basis include, as previously noted, a free resolution of the $\mathcal{O}_{U_{\mu}}[x, y]$-module $\mathcal{O}_{U_{\mu}}[x, y] / \mathcal{J}_{\mu}$, and the recovery of the $g_{r s}$ as the signed maximal minors of the syzygy matrix.

Section 5: An explicit representation of $\mathcal{O}_{U_{\mu}}$ as a quotient ring. Obtains the first set of generators of the kernel $\mathfrak{R}_{\mu}$ of the map $u_{\mu}^{*}(2)$.

Section 6: A second set of generators of the ideal $\mathfrak{R}_{\mu}$. Obtains the second set of generators of the kernel of the map $u_{\mu}^{*}$.

Section 7: Smaller generating sets for $\mathcal{O}_{U_{\mu}}$ and affine cell criteria. Identifies a "small" subset of $\mathfrak{c}_{\mu}$ that generates $\mathcal{O}_{U_{\mu}}$ as a k-algebra, and presents sufficient conditions on $\mu$ for $U_{\mu}$ to be an affine cell in $\mathbf{H}^{n}$.

\section{Hilb ${ }_{\mathrm{A}_{\mathrm{k}}^{2}}^{n}$ and the affine open subschemes $U_{\mu}$.}

In this section of the paper, we briefly recall the definition and some properties of the Hilbert scheme $\operatorname{Hilb}_{\mathrm{A}_{\mathrm{k}}^{2}}^{n}=\mathbf{H}^{n}$, and summarize the necessary background from [7] regarding the open subschemes $U_{\mu} \subseteq \mathbf{H}^{n}$ (defined for every partition $\mu$ of $n$ ) which are the main focus of this paper. Recall that the ground field $k$ is algebraically closed and of arbitrary characteristic.

2.1. Definition of $\mathbf{H}^{n}$. The variety $\mathbf{H}^{n}$ can be defined most naively as the set of ideals $I \subseteq \mathrm{k}[x, y]$ having colength $n$, that is, $\operatorname{dim}_{\mathrm{k}}(\mathrm{k}[x, y] / I)=n$. 
Less naively, the variety $\mathbf{H}^{n}$ parameterizes in a natural way the set of 0 dimensional closed subschemes

$$
Z=\operatorname{Spec}(\mathrm{k}[x, y] / I) \subseteq \mathrm{A}_{\mathrm{k}}^{2}=\operatorname{Spec}(\mathrm{k}[x, y])
$$

that have length $n$. It is defined as the open subscheme of Hilb $\mathrm{P}_{\mathrm{k}}^{n}$ (the existence of which is a consequence of Grothendieck's general construction given in [5]) arising from the inclusion of $A_{k}^{2}$ into $P_{k}^{2}$ as a standard affine. By pullback, $\mathbf{H}^{n}$ inherits a universal closed subscheme $Z_{n} \subseteq \mathbf{H}^{n} \times \mathrm{A}_{\mathrm{k}}^{2}$, which is finite and flat of degree $n$ over $\mathbf{H}^{n}$ via the first projection, and satisfies the following property:

Let $T$ be a separated scheme of finite type over $\mathrm{k}$. Then the set of maps $f: T \rightarrow \mathbf{H}^{n}$ is in natural bijective correspondence with the set of closed subschemes $Z_{f} \subseteq T \times \mathrm{A}_{\mathrm{k}}^{2}$ that are finite and flat of degree $n$ over $T$; the bijection $f \mapsto Z_{f}$ is defined by $Z_{f}=T \times \times_{\mathbf{H}^{n}} Z_{n}$.

In particular, the inclusion of the k-point $t \in \mathbf{H}^{n}$ corresponds to a unique closed subscheme $Z_{t} \subseteq t \times \mathrm{A}_{\mathrm{k}}^{2} \approx \mathrm{A}_{\mathrm{k}}^{2}$; the map $t \mapsto Z_{t}$ defines a bijection from the set of k-points of $\mathbf{H}^{n}$ to the set of 0-dimensional closed subschemes of length $n$ (or, equivalently, to the set of ideals $I \subseteq \mathrm{k}[x, y]$ of colength $n$ ). We often identify $I$ with its associated point in $\mathbf{H}^{n}$, allowing us to write, for example, $I \in \mathbf{H}^{n}$.

2.2. The affine open subschemes $U_{\mu}$. In [7], M. Haiman obtains a finite covering of $\mathbf{H}^{n}$ by affine open subschemes $U_{\mu}$, where $\mu$ runs through the partitions of $n$. Given the partition $\mu=\left(p_{1}, p_{2}, \ldots, p_{\ell}\right)$, with (positive) parts listed in decreasing order, we define the set of $n$ monomials

$$
\mathfrak{B}_{\mu}=\left\{x^{h} y^{k} \mid 0 \leq h<\ell, 0 \leq k<p_{h+1}\right\} .
$$

When displayed as an array with rows indexed by $x$-degree and columns indexed by $y$-degree (starting at 0 in each case), $\mathfrak{B}_{\mu}$ yields a diagram of the partition with the rows representing the parts. An example should suffice to make the idea clear; witness the set $\mathfrak{B}_{(5,3,2,2)}$ :

$$
\begin{array}{ccccc}
x^{3} & x^{3} y & & & \\
x^{2} & x^{2} y & & & \\
x & x y & x y^{2} & & \\
1 & y & y^{2} & y^{3} & y^{4} .
\end{array}
$$

We write $(h, k) \in \mu$ to indicate that the inequalities in (4) are satisfied, that is, that $x^{h} y^{k} \in \mathfrak{B}_{\mu}$. We call the elements of $\mathfrak{B}_{\mu}$ partition monomials.

Following [7, p. 206],

[w]e now define

$$
U_{\mu}=\left\{I \in \mathbf{H}^{n} \mid \mathfrak{B}_{\mu} \text { spans } \mathrm{k}[x, y] / I\right\} .
$$


Here we really mean that [the] image of $\mathfrak{B}_{\mu}$ modulo $I$ spans $\mathrm{k}[x, y] / I$. Of course this makes $\mathfrak{B}_{\mu}$ a basis modulo $I$, since $\operatorname{dim}_{\mathrm{k}}(\mathrm{k}[x, y] / I)=n$. Since $\mathfrak{B}_{\mu}$ is a basis, for each monomial $x^{r} y^{s}$ and ideal $I \in U_{\mu}$ there is a unique expansion

$$
x^{r} y^{s}=\sum_{(h, k) \in \mu} c_{h k}^{r s}(I) x^{h} y^{k} \quad(\bmod I),
$$

whose coefficients depend on $I$ and thus define a collection of functions $c_{h k}^{r s}$ on $U_{\mu}$.

We have:

Proposition 2.2.1 (Haiman [7, Proposition 2.1]). The sets $U_{\mu}$ are open affine subvarieties which cover $\mathbf{H}^{n}$. The affine coordinate ring $\mathcal{O}_{U_{\mu}}$ is generated by the functions $c_{h k}^{r s}$, for $(h, k) \in \mu$ and all $(r, s)$.

[We do not quote the proof.]

2.3. Universal property of $U_{\mu}$. For the proof of Theorem 5.1.1, we need to construct a map with $U_{\mu} \subseteq \mathbf{H}^{n}$ as target. By the universal property of $\mathbf{H}^{n}$, such a map corresponds to a suitable family of subschemes over the source of the map; we make precise one formulation of "suitable family" in Proposition 2.3.2.

Lemma 2.3.1. Let $T$ be a separated scheme of finite type over $\mathrm{k}$, and $f: T \rightarrow \mathbf{H}^{n}$ a map such that for every k-rational point $t \in T$ we have that $f(t) \in U_{\mu}$. Then $f$ factors through the inclusion $U_{\mu} \hookrightarrow \mathbf{H}^{n}$.

Proof. It suffices to show that if $x \in T$ is an arbitrary scheme-theoretic point, then $f(x) \in U_{\mu}$. If not, then $f(\bar{x}) \subseteq \mathbf{H}^{n}-U_{\mu}$; however, since $\mathrm{k}$ is algebraically closed, the k-rational points of $T$ are very dense [6, Corollaire 6.5 .3 , p. 309], whence $\bar{x}$ contains a k-rational point that perforce maps to the complement of $U_{\mu}$ under $f$, a contradiction.

$U_{\mu}$ now inherits the following universal property from $\mathbf{H}^{n}$ :

Proposition 2.3.2. Let $T$ be as in the lemma. Then the set of maps $f: T \rightarrow U_{\mu}$ is in natural bijective correspondence with the set of closed subschemes $Z_{f} \subseteq T \times \mathrm{A}_{\mathrm{k}}^{2}$ that are finite and flat of degree $n$ over $T$, and such that the fiber over every $\mathrm{k}$-point $t \in T$ is cut out by an ideal $I_{t} \subseteq \mathrm{k}[x, y]$ having the set of partition monomials $\mathfrak{B}_{\mu}$ as a $\mathrm{k}$-basis of the quotient $\mathrm{k}[x, y] / I_{t}$ (briefly, $I_{t} \in U_{\mu}$ ).

Proof. A closed subscheme $Z_{f}$ as described in the proposition is uniquely associated with a map $f: T \rightarrow \mathbf{H}^{n}$ by the universal property of $\mathbf{H}^{n}$ (3), and this map must factor through $U_{\mu}$ by the lemma. It is clear that the correspondence $Z_{f} \leftrightarrow f$ is bijective, and that $Z_{f}$ is obtained as the pullback 
of the restriction to $U_{\mu} \times \mathrm{A}_{\mathrm{k}}^{2}$ of the universal closed subscheme $Z_{n} \subseteq \mathbf{H}^{n} \times$ $\mathrm{A}_{\mathrm{k}}^{2}$.

2.4. Nonsingularity of $\mathbf{H}^{n}$. It is well-known that $\mathrm{Hilb}_{X}^{n}$ is irreducible and nonsingular whenever $X$ is an irreducible and nonsingular surface [4]. Haiman gives a delightful proof that these facts hold for $\mathbf{H}^{n}[\mathbf{7}$, Proposition 2.4, pp. 208-211]. The proof begins with the observation that

[t]he two-dimensional torus group

$$
\mathbf{T}^{2}=\left\{(t, q) \mid t, q \in \mathbf{k}^{*}\right\}
$$

acts algebraically on $\mathrm{A}_{\mathrm{k}}^{2}$ by $(t, q) \cdot(\xi, \zeta)=(t \xi, q \zeta)$, or equivalently on $\mathrm{k}[x, y]$ by $(t, q) \cdot x=t x,(t, q) \cdot y=q y$. There is an induced action on $\mathbf{H}^{n}$ which, since (7) must remain invariant, is given by $(t, q) \cdot c_{h k}^{r s}=t^{r-h} q^{s-k} c_{h k}^{r s}$. One must take care in computing $(t, q) \cdot I$ for $I \in \mathbf{H}^{n}$ to remember that this means the pullback of $I$ via the homomorphism $(t, q): \mathrm{k}[x, y] \rightarrow \mathrm{k}[x, y]$, given by $(t, q) \cdot I=\left\{p\left(t^{-1} x, q^{-1} y\right) \mid p(x, y) \in I\right\}$.

Haiman observes that the $\mathbf{T}^{2}$ fixed points of $\mathbf{H}^{n}$ are exactly the points corresponding to monomial ideals. Every monomial ideal has the form

$$
I_{\mu}=\left(\left\{x^{p} y^{q} \mid(p, q) \notin \mu\right\}\right)
$$

for some partition $\mu$ of $n$, and every partition $\mu$ gives rise to a monomial ideal in this way. Note that the subscheme

$$
Z_{\mu}=\operatorname{Spec}\left(\mathrm{k}[x, y] / I_{\mu}\right)
$$

is concentrated at the origin of $\mathrm{A}_{\mathrm{k}}^{2}$, and that $I_{\mu} \in U_{\mu}$. Haiman proves [7, Lemma 2.3, p. 209] that every ideal $I \in \mathbf{H}^{n}$ has a torus fixed point in the closure of its orbit (in fact, the initial ideal of $I$ for the lexicographic monomial ordering with $y>x$ is a monomial ideal with this property). Since the singular locus of $\mathbf{H}^{n}$ is closed and $\mathbf{T}^{2}$-stable, it must either be empty or contain a monomial ideal. Therefore, to prove that $\mathbf{H}^{n}$ is nonsingular, it suffices to show that each monomial ideal in $\mathbf{H}^{n}$ is a nonsingular point. Haiman does this by explicitly constructing local parameters at each such point $I_{\mu}$. In his words [7, p. 210],

[t]he maximal ideal $\mathfrak{m}$ of $I_{\mu}$ in $\mathcal{O}_{U_{\mu}}$ is given by

$$
\mathfrak{m}=\left(\left\{c_{h k}^{r s} \mid(h, k) \in \mu,(r, s) \notin \mu\right\}\right) .
$$

(For $(r, s) \in \mu$, we have $c_{h k}^{r s}=0$ identically for $(h, k) \neq(r, s)$, and $c_{r s}^{r s}=1$, so we omit these $c_{h k}^{r s}$ from the ideal.) ... [Consider now the diagram of the monomials in $\mathfrak{B}_{\mu}$, as in (5).] We single out two special coordinate functions $c_{h k}^{r s}$ for each 
$(h, k) \in \mu$. Let $(f, k)$ be the top [entry] in column $k$ and let $(h, g)$ be the last [entry] in row $h$. This given, let

$$
\begin{aligned}
u_{h, k} & =c_{f, k}^{h, g+1}, \\
d_{h, k} & =c_{h, g}^{f+1, k} .
\end{aligned}
$$

These will be our spanning parameters for $\mathfrak{m} / \mathfrak{m}^{2}$.

For example, when $\mu=(5,3,2,2)$, as in $(5)$, and $(h, k)=(0,0)$, we have that

$$
u_{0,0}=c_{3,0}^{0,5} \text { and } d_{0,0}=c_{0,4}^{4,0}
$$

it is a good exercise to list the remaining 22 spanning parameters in this case.

Haiman's proof that the functions (10) span $\mathfrak{m} / \mathfrak{m}^{2}$ over $k$ proceeds as follows [7, pp. 210-211]:

Multiplying (7) through by $x$, then expanding each term on the right by (7) again and comparing coefficients yields the identity

$$
c_{h, k}^{r+1, s}=\sum_{\left(h^{\prime}, k^{\prime}\right) \in \mu} c_{h^{\prime} k^{\prime}}^{r s} c_{h k}^{h^{\prime}+1, k^{\prime}}
$$

for all $(h, k) \in \mu$ and all $(r, s)$. Proceeding similarly with $y$ in place of $x$ yields

$$
c_{h, k}^{r, s+1}=\sum_{\left(h^{\prime}, k^{\prime}\right) \in \mu} c_{h^{\prime} k^{\prime}}^{r s} c_{h k}^{h^{\prime}, k^{\prime}+1} .
$$

Modulo $\mathfrak{m}^{2}$, the terms $c_{h^{\prime} k^{\prime}}^{r s} c_{h k}^{h^{\prime}+1, k^{\prime}}$ on the right-hand side of (11) reduce to zero for $\left(h^{\prime}+1, k^{\prime}\right) \notin \mu$ and for $\left(h^{\prime}+1, k^{\prime}\right) \in \mu$, $\left(h^{\prime}+1, k^{\prime}\right) \neq(h, k)$ [here we are assuming that $(r, s) \notin \mu$, so that $\left.c_{h k}^{r s} \in \mathfrak{m}\right]$. The remaining term is $c_{h-1, k}^{r s}$, or zero if $h=$ 0 . Corresponding reductions apply to the right-hand side of (12). Thus in $\mathfrak{m} / \mathfrak{m}^{2}$ we have

$$
\begin{array}{ll}
c_{h k}^{r+1, s}=c_{h-1, k}^{r s}, & \text { or } 0 \text { if } h=0 ; \\
c_{h k}^{r, s+1}=c_{h, k-1}^{r s}, & \text { or } 0 \text { if } k=0 .
\end{array}
$$

It is convenient to depict each $c_{h k}^{r s}$ by an arrow from $(r, s)$ to $(h, k)$, as shown [in Figure 2]: Equations (13) say that we may move these arrows horizontally or vertically without changing their values modulo $\mathrm{m}^{2}$, provided we keep the head inside $\mu$ and the tail outside. More generally, as long as we keep the tail in the first quadrant and outside $\mu$, we may even move the head across the $x$ - or $y$-axis. When this is 


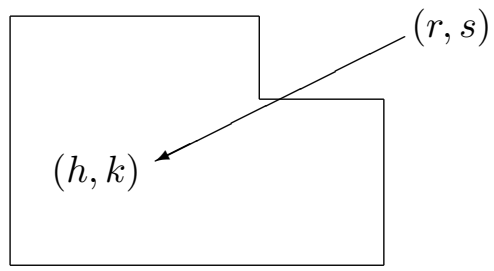

Figure 2. $c_{h k}^{r s}$ represented as an arrow.

possible, the value of the arrow is zero. [The passage goes on to verify that any arrow representing one of the generators of $\mathfrak{m}$ (9) can be translated horizontally and/or vertically (mod $\mathfrak{m}^{2}$ ) until it has been shown either to be zero or to coincide with one of the functions (10); whence, the latter $\operatorname{span} \mathfrak{m} / \mathfrak{m}^{2}$, and therefore constitute a set of local parameters at $I_{\mu}$.]

2.5. Local parameters at every point of $\mathbf{H}^{n}$. Haiman's construction of local parameters at monomial ideals in fact yields explicit local parameters at every ideal $I \in \mathbf{H}^{n}$. Given $I$, we begin by computing the initial ideal $\operatorname{in}(I)=I_{\mu}$ for the lexicographic monomial ordering with $y>x$. We write

$$
\mathrm{p}_{\mu}=\left\{u_{h, k}, d_{h, k} \mid(h, k) \in \mu\right\}
$$

for the set of local parameters (10) at $I_{\mu}$, which is accordingly a $k$-algebraically independent set; the inclusion $\mathrm{k}\left[\mathrm{p}_{\mu}\right] \subseteq \mathcal{O}_{U_{\mu}}$ yields the morphism

$$
\varepsilon_{\mu}: U_{\mu} \rightarrow \operatorname{Spec}\left(\mathrm{k}\left[\mathrm{p}_{\mu}\right]\right)=\mathrm{A}_{\mathrm{k}}^{2 n} .
$$

We have the following:

Lemma 2.5.1. The map $\varepsilon_{\mu}$ is scheme-theoretically dominant $[6,5.4$, p. 283] and étale at $I_{\mu} \in U_{\mu}$.

Proof. The first assertion follows at once from the definition and the injectivity of the map $\varepsilon_{\mu}^{*}: \mathrm{k}\left[\mathrm{p}_{\mu}\right] \rightarrow \mathcal{O}_{U_{\mu}}$. The second assertion follows from $[\mathbf{1}$, Corollary 4.5, p. 116], since the induced map on completions $\varepsilon_{\mu^{*}}: \hat{O}_{0} \rightarrow \hat{O}_{I_{\mu}}$ is the identity $\mathrm{k}\left[\left[\mathrm{p}_{\mu}\right]\right] \rightarrow \mathrm{k}\left[\left[\mathrm{p}_{\mu}\right]\right]$.

We claim that $\varepsilon_{\mu}$ is étale at $I$. To see this, observe first of all that $\operatorname{Spec}\left(\mathrm{k}\left[\mathrm{p}_{\mu}\right]\right)=\mathrm{A}_{\mathrm{k}}^{2 n}$ inherits a $\mathbf{T}^{2}$-action from $\mathbf{H}^{n}$; for $(t, q) \in \mathbf{T}^{2}$, the comorphism of the map

$$
(t, q): \operatorname{Spec}\left(\mathrm{k}\left[\mathrm{p}_{\mu}\right]\right) \rightarrow \operatorname{Spec}\left(\mathrm{k}\left[\mathrm{p}_{\mu}\right]\right)
$$

is defined by $(t, q) \cdot c_{h k}^{r s}=t^{r-h} q^{s-k} c_{h k}^{r s}$ for $c_{h k}^{r s} \in \mathrm{p}_{\mu}$. In other words, the action is defined by restricting the comorphism of $(t, q): U_{\mu} \rightarrow U_{\mu}$ to $\mathrm{k}\left[\mathrm{p}_{\mu}\right] \subseteq \mathcal{O}_{U_{\mu}}$; it follows at once that the left-hand diagram in Figure 3 is commutative for all $(t, q) \in \mathbf{T}^{2}$. 

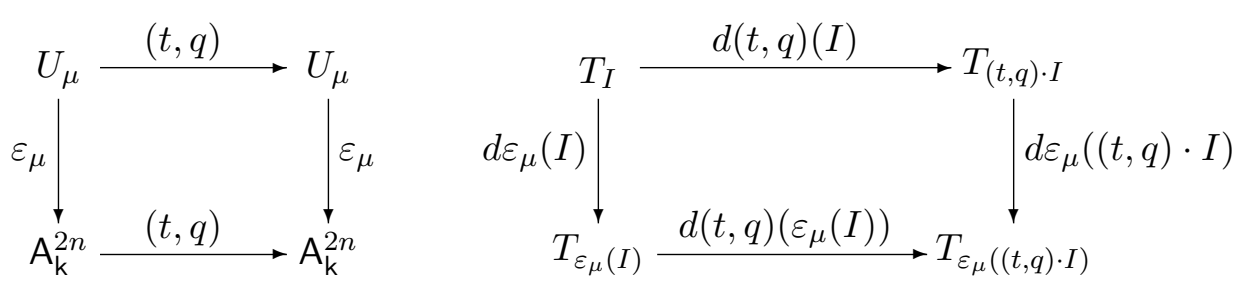

Figure 3. Commutative diagrams associated with $\varepsilon_{\mu}$.

The right-hand square of tangent spaces and k-linear maps in Figure 3 is induced by the left-hand square; to show that $\varepsilon_{\mu}$ is étale at $I$, we must show that the map $d \varepsilon_{\mu}(I)$ is an isomorphism. However, since $\varepsilon_{\mu}$ is étale at $I_{\mu}$, and therefore in a neighborhood thereof (see, e.g., [1, Proposition 4.6, p. 116]), and since $I_{\mu}$ lies in the closure of the $\mathbf{T}^{2}$-orbit of $I$, as stated in Section 2.4, we can choose $(t, q) \in \mathbf{T}^{2}$ so that $d \varepsilon_{\mu}((t, q) \cdot I)$ is an isomorphism. Since the horizontal arrows are clearly isomorphisms, we conclude that $d \varepsilon_{\mu}(I)$ is an isomorphism, as desired. We restate our conclusion as:

Proposition 2.5.2. Let $I \in \mathbf{H}^{n}$ and let $I_{\mu}$ be the initial ideal of I for the lexicographic monomial order with $y>x$. Then the set of functions

$$
\left\{c_{h k}^{r s}-c_{h k}^{r s}(I) \mid c_{h k}^{r s} \in \mathrm{p}_{\mu}\right\}
$$

is a set of local parameters at $I \in \mathbf{H}^{n}$.

In the next section, we exhibit a certain finite subset $\mathfrak{c}_{\mu}$ of the (infinite set of) $c_{h k}^{r s}$ that generates $\mathcal{O}_{U_{\mu}}$ as a k-algebra; the set $\mathfrak{c}_{\mu}$ contains the set $\mathrm{p}_{\mu}$ of local parameters at $I_{\mu}$. (Please note that the subset $\mathfrak{c}_{\mu}$ is chosen for convenience; it is typically far from a minimal generating set.) In order to prove that $\mathfrak{c}_{\mu}$ does in fact generate $\mathcal{O}_{U_{\mu}}$, we need the relations (11), (12). Later, in Section 7 , we show that $\mathcal{O}_{U_{\mu}}$ is in fact generated by a subset ex ${ }_{\mu} \subseteq$ $\mathfrak{c}_{\mu}$ that also contains $\mathrm{p}_{\mu}$; the subset $\mathrm{ex}_{\mu}$ is typically much smaller than $\mathfrak{c}_{\mu}$ (see (58) and the following example), but again may not be a minimal generating set. In certain special cases, one has either that $\mathrm{p}_{\mu}=\mathrm{ex}_{\mu}$ (Section 7.3) or that $\mathrm{ex}_{\mu} \subseteq \mathrm{k}\left[\mathrm{p}_{\mu}\right]$ (Section 7.4, where the relations (11), (12) again play a key role), from which follows that $\mathcal{O}_{U_{\mu}}$ is the polynomial ring $\mathrm{k}\left[\mathrm{p}_{\mu}\right]$, or, equivalently, that the map $\varepsilon_{\mu}$ (15) is an isomorphism. Of course, whenever $\mathrm{p}_{\mu}$ generates $\mathcal{O}_{U_{\mu}}$, it is a minimal generating set (since it has cardinality $2 n$ equal to the dimension of $U_{\mu}$ ), but there are partitions $\mu$ for which $\mathrm{p}_{\mu}$ fails to generate $\mathcal{O}_{U_{\mu}}$. I do not know how to find a minimal generating set in all cases.

\section{A finite set of generators of $\mathcal{O}_{U_{\mu}}$.}

We have two objectives in this section: The first is to demonstrate that a certain finite subset $\mathfrak{c}_{\mu}$ of the functions $c_{h k}^{r s}$ generates the affine coordinate 
ring $\mathcal{O}_{U_{\mu}}$ as a k-algebra. The second is to show that certain polynomials associated to the set $\mathfrak{c}_{\mu}$ form a basis for the ideal

$$
\mathfrak{I}_{\mu} \subseteq O_{U_{\mu}}[x, y]
$$

that cuts out the universal closed subscheme over $U_{\mu}$.

3.1. Leading monomials and generators of $\mathcal{O}_{U_{\mu}}$. We begin with a partition $\mu=\left(p_{1}, p_{2}, \ldots, p_{\ell}\right)$ of $n$ (with parts listed in decreasing order) which we view as the set of partition monomials $\mathfrak{B}_{\mu}$ (4) arranged in rows and columns as in (5). We write

$$
\begin{aligned}
\ell & =\text { the number of parts of } \mu \\
& =\text { the number of rows of } \mathfrak{B}_{\mu} ; \\
d_{\mu} & =\text { the number of distinct parts of } \mu ; \\
\mu(i) & =\text { the number of times the integer } i \text { occurs in } \mu ; \\
p_{1} & =\text { the largest part in } \mu \\
& =\text { the number of columns of } \mathfrak{B}_{\mu} ; \\
p_{\ell} & =\text { the smallest part in } \mu .
\end{aligned}
$$

We say that a monomial $x^{r} y^{s}$ is a leading monomial of $\mu$ if it lies on the "boundary" of $\mathfrak{B}_{\mu}$ - either immediately above a column or immediately to the right of a row. For example, when $\mu=(5,3,2,2)$, the leading monomials are shown in bold in Figure 4. We call the leading monomials situated

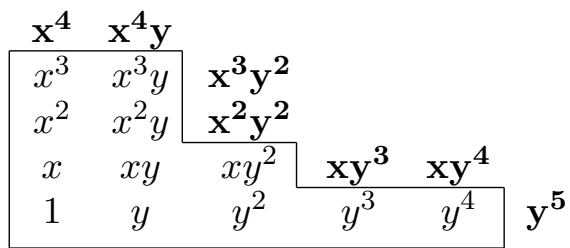

Figure 4. The partition monomials for $\mu=(5,3,2,2)$ enclosed in a box, with the leading monomials shown in boldface. In this case, $\ell=4, d_{\mu}=3, \mu(2)=2, \mu(3)=1, \mu(5)=$ $1, p_{1}=5$, and $p_{\ell}=p_{4}=2$.

above the columns (resp. to the right of the rows) of $\mathfrak{B}_{\mu}$ the top (resp. side) monomials associated to $\mu$. Because $d_{\mu}-1$ of the leading monomials (those in the "notch" positions $-x^{2} y^{2}$ and $x=y^{3}$ in Figure 4) are both top and side monomials, we have in general that

$$
\begin{aligned}
\Lambda_{\mu} & =\text { the number of leading monomials } \\
& =(\# \text { top monomials })+(\# \text { side monomials })-\left(d_{\mu}-1\right) \\
& =p_{1}+\ell-d_{\mu}+1 .
\end{aligned}
$$


We index the leading monomials $x^{r_{j}} y^{s_{j}}$ from $j=1$ to $\Lambda_{\mu}$ by starting at the upper left of the diagram of $\mu$ and traversing the boundary in a clockwise fashion. In the example, this yields the sequence

$$
x^{4}, x^{4} y, x^{3} y^{2}, x^{2} y^{2}, x y^{3}, x y^{4}, y^{5} .
$$

Remark 3.1.1. Observe that the first (resp. last) leading monomial in the sequence is $x^{r_{1}}=x^{\ell}\left(\operatorname{resp} . y^{s_{\Lambda_{\mu}}}=y^{p_{1}}\right)$.

For $I \in U$, form the expansion (7) for each of the leading monomials $x^{r_{j}} y^{s_{j}}, 1 \leq j \leq \Lambda_{\mu}$, to obtain

$$
x^{r_{j}} y^{s_{j}}=\sum_{(h, k) \in \mu} c_{h k}^{r_{j}, s_{j}}(I) x^{h} y^{k} \quad(\bmod I) ;
$$

recall that the coefficients can be viewed as functions on $U_{\mu}$. Our generating set $\mathfrak{c}_{\mu}$ consists of all the coefficient functions appearing in (18), that is,

$$
\mathfrak{c}_{\mu}=\left\{c_{h k}^{r_{j}, s_{j}} \mid 1 \leq j \leq \Lambda_{\mu},(h, k) \in \mu\right\} ;
$$

note that the cardinality of of $\mathfrak{c}_{\mu}$ is

$$
\left|\mathfrak{c}_{\mu}\right|=n \cdot \Lambda_{\mu},
$$

and that $\mathrm{p}_{\mu} \subseteq \mathfrak{c}_{\mu}$ (immediate from the definition of $\mathrm{p}_{\mu}(14)$ ). Of course, we should not refer to $\mathfrak{c}_{\mu}$ as a generating set until we have proven it so:

Proposition 3.1.2. The set $\mathfrak{c}_{\mu}$ generates the affine coordinate ring $\mathcal{O}_{U_{\mu}}$ as an algebra over $\mathrm{k}$.

Proof. By Proposition 2.2.1, the ring $\mathcal{O}_{U_{\mu}}$ is generated by the set of all $c_{h k}^{r s}$, so it suffices to show that the subset $\mathfrak{c}_{\mu}$ generates every $c_{h k}^{r s}$. In fact, we only need to do this for $(r, s) \notin \mu$, since, as was observed following $(9), c_{h k}^{r s}$ is identically either 0 or 1 if $(r, s) \in \mu$. We say that the pair $(r, s) \notin \mu$ is covered if the function $c_{h k}^{r s}$ is in the subring of $\mathcal{O}_{U_{\mu}}$ generated by $\mathfrak{c}_{\mu}$ for all $(h, k) \in \mu$. We must show that every pair $(r, s) \notin \mu$ is covered; it is clear at the outset that this is so for each of the pairs $\left(r_{j}, s_{j}\right)$ associated to the leading monomials.

The relation (11) implies that the monomial $x^{r} y^{s}$ is covered provided that

1) its "downstairs" neighbor $x^{r-1} y^{s}$ is covered, and

2) every pair $(h+1, k) \notin \mu$, with $(h, k) \in \mu$, is covered.

However, the pairs $(h+1, k)$ in item 2 are exactly the pairs $\left(r_{j}, s_{j}\right)$ associated to the top leading monomials (see Figure 4), and we know that all such pairs are covered. Therefore, whenever $(r, s)$ is covered, we can proceed inductively to conclude that $(r+1, s),(r+2, s), \ldots$, are covered as well. Similarly, using relation (12) and working horizontally rather than vertically, we see that whenever $(r, s)$ is covered, so too are $(r, s+1),(r, s+2), \ldots$. Since it is clear that every pair $(r, s) \notin \mu$ can be reached by traversing a path 
beginning at a leading monomial and consisting of vertical and/or horizontal segments, it follows that every such pair $(r, s)$ is covered, as desired.

Remark 3.1.3. Recall that, as stated at the end of Section 2, the generating set given by Proposition 3.1.2 is typically far from minimal. For example, the proof of the proposition can be modified to show that the subset

$$
\left\{c_{h k}^{r_{j}, s_{j}} \mid x^{r_{j}} y^{s_{j}} \text { is either a top monomial or } y^{p_{1}},(h, k) \in \mu\right\} \subseteq \mathfrak{c}_{\mu}
$$

also generates $\mathcal{O}_{U_{\mu}}$; this subset is a proper subset of $\mathfrak{c}_{\mu}$ whenever there is at least one side monomial $x^{r_{j}} y^{s_{j}}$ with $j<\Lambda_{\mu}$ that is not also a top monomial (such as $x^{3} y^{2}$ in Figure 4). At the moment, we can assert that a set of k-algebra generators of $\mathcal{O}_{U_{\mu}}$ of minimal cardinality must have at least $2 n$ members (for reasons of dimension), and can have at most $\Lambda_{\mu} n$ members (by (20)); we will tighten the upper bound in Section 7 .

Remark 3.1.4. Recalling (9), it is clear that the monomial ideal $I_{\mu} \subseteq$ $k[x, y](8)$ is the point of $U_{\mu}$ at which all the functions in our generating set $\mathfrak{c}_{\mu}$ vanish.

3.2. The ideal of the universal closed subscheme. We now consider the restriction of the universal closed subscheme $Z_{n} \subseteq \mathbf{H}^{n} \times \mathrm{A}_{\mathrm{k}}^{2}$ (see Section 2.1 and the proof of Proposition 2.3.2) to $U_{\mu}$; we denote the restricted subscheme by $Z_{U_{\mu}}$, and write

$$
Z_{U_{\mu}}=\operatorname{Spec}\left(\mathcal{O}_{U_{\mu}}[x, y] / \mathfrak{I}_{\mu}\right) .
$$

By definition, the ring $\mathcal{O}_{U_{\mu}}[x, y] / \mathfrak{I}_{\mu}$ is finite and flat of degree $n$ over $\mathcal{O}_{U_{\mu}}$. Moreover, for any point $I \in U_{\mu}$, given as a map $i$ : $\operatorname{Spec}(\mathrm{k}) \rightarrow U_{\mu}$ with comorphism $i^{*}: \mathcal{O}_{U_{\mu}} \rightarrow \mathrm{k}$, we have that

$$
\mathrm{k} \otimes_{\mathcal{O}_{U_{\mu}}} \mathcal{O}_{U_{\mu}}[x, y] / \mathfrak{I}_{\mu}=\mathrm{k}[x, y] / I
$$

that is, $\mathfrak{I}_{\mu}$ extends to $I$ under the map $\mathcal{O}_{U_{\mu}}[x, y] \rightarrow \mathrm{k}[x, y]$ induced by $i^{*}$. We seek to exhibit a basis of the ideal $\mathfrak{I}_{\mu}$; to this end, we define, for each leading monomial $x^{r_{j}} y^{s_{j}}$, the polynomial

$$
g_{j}=x^{r_{j}} y^{s_{j}}-\sum_{(h, k) \in \mu} c_{h k}^{r_{j}, s_{j}} x^{h} y^{k} \in \mathcal{O}_{U_{\mu}}[x, y] .
$$

If the leading monomial $x^{r_{j}} y^{s_{j}}$ is a top (resp. side) monomial of $\mu$, then by extension we refer to $g_{j}$ as a top (resp. side) polynomial of $\mu$.

Proposition 3.2.1. The ideal $\mathfrak{I}_{\mu}$ is generated by the $g_{j}, 1 \leq j \leq \Lambda_{\mu}$; in symbols, $\mathfrak{I}_{\mu}=\left(g_{1}, g_{2}, \ldots, g_{\Lambda_{\mu}}\right)$.

For the proof, we need two lemmas.

Lemma 3.2.2. The quotient $\mathcal{O}_{U_{\mu}}[x, y] / \mathfrak{I}_{\mu}$ is free of rank $n$ over $\mathcal{O}_{U_{\mu}}$, with the (image of the) set $\mathfrak{B}_{\mu}$ of partition monomials constituting a basis. 
Proof. We have already noted that $\mathcal{O}_{U_{\mu}}[x, y] / \mathfrak{I}_{\mu}=Q$ is finite and flat of degree $n$ over $\mathcal{O}_{U_{\mu}}=A$; whence, the sheaf $\widetilde{Q}$ is locally free of degree $n$ on $U_{\mu}=\operatorname{Spec}(A)$. By definition of $U_{\mu}(6)$, (the image of) $\mathfrak{B}_{\mu}$ yields a basis of $\mathrm{k}[x, y] / I$ for every $I \in U_{\mu}$; it follows from Nakayama's lemma that $\mathfrak{B}_{\mu}$ generates (and therefore gives a basis of) $\widetilde{Q}$ in a local neighborhood of every point of $U_{\mu}$. Consequently, the map $A^{n} \rightarrow Q$ induced by the $n$-element set $\mathfrak{B}_{\mu}$ localizes to an isomorphism everywhere on $U_{\mu}$, and is therefore itself an isomorphism.

Lemma 3.2.3. The quotient $\mathcal{O}_{U_{\mu}}[x, y] /\left(g_{1}, g_{2}, \ldots, g_{\Lambda_{\mu}}\right)$ is generated as an $\mathcal{O}_{U_{\mu}}$-module by the (images of the) partition monomials.

Proof. We write $\left(g_{1}, g_{2}, \ldots, g_{\Lambda_{\mu}}\right)=\mathfrak{G}$ and, as before, $\mathcal{O}_{U_{\mu}}=A$. It suffices to prove that every monomial in $x$ and $y$ is congruent $(\bmod \mathfrak{G})$ to an $A$-linear combination of partition monomials. This is immediate for the partition monomials themselves, and is also clearly true for the leading monomials, by definition of the $g_{j}$. We may therefore proceed by induction on the total degree of a monomial, the base case having already been checked since $x^{0} y^{0}=1$ is always a partition monomial.

Suppose therefore that every monomial of total degree $<r+s$ is congruent $(\bmod \mathfrak{G})$ to an $A$-linear combination of partition monomials, and consider the monomial $x^{r} y^{s}$. If $r>0$, we have that $x^{r} y^{s}=x \cdot x^{r-1} y^{s}$; whence, by the induction hypothesis,

$$
\begin{aligned}
x^{r} y^{s}=x \cdot x^{r-1} y^{s} & \equiv x \cdot\left(\sum_{(h, k) \in \mu} a_{h k}^{r-1, s} x^{h} y^{k}\right) \\
& \equiv \sum_{(h, k) \in \mu} a_{h k}^{r-1, s} x^{(h+1)} y^{k}(\bmod \mathfrak{G}),
\end{aligned}
$$

where the coefficients $a_{h k}^{r-1, s} \in A$. If any of the monomials $x^{h+1} y^{k}$ in the last sum are not partition monomials, then they are top leading monomials, and can accordingly be expanded $(\bmod \mathfrak{G})$ as $A$-linear combinations of partition monomials, showing that such an expansion also obtains for $x^{r} y^{s}$, as desired. If $r=0$, then $s>0$, and the proof is similar.

Proof of Proposition 3.2.1. We first show that $g_{j} \in \mathfrak{I}_{\mu}$ for $1 \leq j \leq \Lambda_{\mu}$. By Lemma 3.2.2, we have that each leading monomial $x^{r_{j}} y^{s_{j}}$ is congruent $\left(\bmod \mathfrak{I}_{\mu}\right)$ to a unique $\mathcal{O}_{U_{\mu}}$-linear combination of partition monomials. Put another way, $\mathfrak{I}_{\mu}$ contains $\Lambda_{\mu}$ uniquely determined polynomials of the form

$$
x^{r_{j}} y^{s_{j}}-\sum_{(h, k) \in \mu} d_{h k}^{r_{j}, s_{j}} x^{h} y^{k}, 1 \leq j \leq \Lambda_{\mu}, d_{h k}^{r_{j}, s_{j}} \in A .
$$

Specializing to any point $I \in U_{\mu}$, we see that $c_{h k}^{r_{j}, s_{j}}(I)=d_{h k}^{r_{j}, s_{j}}(I)$ must hold; in other words, the functions $c_{h k}^{r_{j}, s_{j}}$ and $d_{h k}^{r_{j}, s_{j}}$ have the same k-values at every point. Since $\mathcal{O}_{U_{\mu}}$ is an integral domain (in particular, reduced), we conclude 
that

$$
c_{h k}^{r_{j}, s_{j}}=d_{h k}^{r_{j}, s_{j}}, 1 \leq j \leq \Lambda_{\mu},(h, k) \in \mu,
$$

which implies that the two sets of polynomials (24) and (23) are the same. It follows that each $g_{j}$ is in $\mathfrak{I}$, as desired; whence,

$$
\left(g_{1}, g_{2}, \ldots, g_{\Lambda_{\mu}}\right)=\mathfrak{G} \subseteq \mathfrak{I}_{\mu} .
$$

Now set $Q^{\prime}=\mathcal{O}_{U_{\mu}}[x, y] / \mathfrak{G}$, and, as before, $A=\mathcal{O}_{U_{\mu}}$ and $Q=A[x, y] / \mathfrak{I}_{\mu}$. Since $\mathfrak{G}$ maps to 0 under the quotient map $A[x, y] \rightarrow Q$, we obtain an $A$ linear surjection $\alpha: Q^{\prime} \rightarrow Q$ which maps the coset $x^{h} y^{k}+\mathfrak{G}$ to the coset $x^{h} y^{k}+\mathfrak{I}_{\mu}$ for all $(h, k) \in \mu$. By Lemma 3.2.2, we obtain an $A$-linear map $\beta: Q \rightarrow Q^{\prime}$ by sending $x^{h} y^{k}+\mathfrak{I}_{\mu}$ to $x^{h} y^{k}+\mathfrak{G}$ and extending linearly. Since the cosets $x^{h} y^{k}+\mathfrak{G}$ generate $Q^{\prime}$ as an $A$-module, by Lemma 3.2.3, and are mapped to themselves by the composition $\beta \circ \alpha$, we have that $\beta \circ \alpha$ is the identity map; whence $\alpha$ is injective (as well as surjective), and therefore an isomorphism. It follows at once that $\mathfrak{G}=\mathfrak{I}_{\mu}$, which is the desired conclusion.

Remark 3.2.4. The generating set of $\mathfrak{I}_{\mu}$ given by Proposition 3.2 .1 is not in general minimal. Indeed, it can be shown that the subset

$$
\left\{g_{j} \mid g_{j} \text { is a top polynomial or } g_{\Lambda_{\mu}}\right\} \subseteq\left\{g_{1}, \ldots, g_{\Lambda_{\mu}}\right\}
$$

is a generating set. For example, consider the partition shown in Figure 4. The displayed subset of $\left\{g_{1}, g_{2}, \ldots, g_{\Lambda_{\mu}}\right\}$ omits only the polynomial with leading term $x^{3} y^{2}$; let $\mathfrak{G}^{\prime} \subseteq \mathfrak{I}_{\mu}$ denote the ideal generated by this subset. By multiplying the polynomial with leading term $x^{2} y^{2}$ by $x$ and adding appropriate multiples of the $g$ 's with top leading monomials, we see that the ideal $\mathfrak{G}^{\prime}$ contains a polynomial of the form

$$
x^{3} y^{2}-\sum_{(h, k) \in \mu} d_{h, k} x^{h} y^{k}, d_{h, k} \in \mathcal{O}_{U_{\mu}}
$$

which, lying in $\mathfrak{I}_{\mu}$, must in fact equal the omitted $g$ polynomial with leading monomial $x^{3} y^{2}$ (the unique polynomial of its form in $\mathfrak{I}_{\mu}$ ). It follows that $\mathfrak{I}_{\mu}=\mathfrak{G}^{\prime}$.

In view of (22), Proposition 3.2.1 yields:

Corollary 3.2.5. For $I \in U_{\mu}$, we have that

$$
I=\left(g_{1}(I), g_{2}(I), \ldots, g_{\Lambda_{\mu}}(I)\right) \subseteq \mathrm{k}[x, y],
$$

where $g_{j}(I)$ denotes the polynomial obtained from $g_{j}$ by replacing each coefficient function $c_{h k}^{r_{j}, s_{j}}$ by its value $c_{h k}^{r_{j}, s_{j}}(I)$ at the point $I$.

In the next section, we present a basis of the first syzygy module of $\left(g_{1}, g_{2}, \ldots, g_{\Lambda_{\mu}}\right)$, which leads to a free resolution of the $\mathcal{O}_{U_{\mu}}$-module $\mathcal{O}_{U_{\mu}}[x, y] / \mathfrak{I}_{\mu}$. 


\section{A free resolution of $\mathcal{O}_{U_{\mu}}[x, y] / \mathfrak{I}_{\mu}$.}

In this section we study the relations among the generators $g_{j}(23)$ of the ideal $\mathfrak{I}_{\mu}$ (21). We present an algorithm that yields a basis for the first syzygy module of these generators. (In preparation for subsequent sections, we study the syzygy algorithm in greater generality than is required for the purposes of this section.) As a corollary, we obtain a free resolution of the $\mathcal{O}_{U_{\mu}}$-module $\mathcal{O}_{U_{\mu}}[x, y] / \mathfrak{I}_{\mu}$. We then show that the $g_{j}$ are recovered (up to sign) as the maximal minors of the matrix whose rows are the elements of the syzygy basis.

4.1. Syzygies of $\left(g_{1}, \ldots, g_{\Lambda_{\mu}}\right)$. Recall that a syzygy of $\left(g_{1}, \ldots, g_{\Lambda_{\mu}}\right)=\left(g_{j}\right)$ is a $\left(\Lambda_{\mu}\right)$-tuple $\left(f_{1}, \ldots, f_{\Lambda_{\mu}}\right)$ of elements of $\mathcal{O}_{U_{\mu}}[x, y]$ such that $\sum_{j=1}^{\Lambda_{\mu}}\left(f_{j} \cdot g_{j}\right)$ $=0$; the set of all such syzygies is a submodule $\operatorname{Syz}_{\mu} \subseteq\left(\mathcal{O}_{U_{\mu}}[x, y]\right)^{\Lambda_{\mu}}$, the first syzygy module of $\left(g_{j}\right)$. We will show that this syzygy module is free of rank $\Lambda_{\mu}-1$, with an easily-obtained basis. The key observation for finding syzygies is the following:

Lemma 4.1.1. If $\left(f_{1}, \ldots, f_{\Lambda_{\mu}}\right) \in\left(\mathcal{O}_{U_{\mu}}[x, y]\right)^{\Lambda_{\mu}}$ is such that the polynomial $\sum_{j=1}^{\Lambda_{\mu}}\left(f_{j} \cdot g_{j}\right)$ is an $\mathcal{O}_{U_{\mu}}$-linear combination of partition monomials, then in fact $\left(f_{1}, \ldots, f_{\Lambda_{\mu}}\right)=\left(f_{j}\right)$ is a syzygy of $\left(g_{j}\right)$.

Proof. The polynomial $L=\sum_{j=1}^{\Lambda_{\mu}}\left(f_{j} \cdot g_{j}\right)$ lies in the ideal $\mathfrak{I}_{\mu}$ generated by the $g_{j}$, and so $L \equiv 0\left(\bmod \mathfrak{I}_{\mu}\right)$. But by Lemma $3.2 .2, \mathcal{O}_{U_{\mu}}[x, y] / \mathfrak{I}_{\mu}$ is free over $\mathcal{O}_{U_{\mu}}$ with the partition monomials constituting a basis. Therefore, the only $\mathcal{O}_{U_{\mu}}$-linear combination of the partition monomials that $L$ could equal is the trivial one, which implies that $\left(f_{j}\right)$ is a syzygy of $\left(g_{j}\right)$.

The basic idea is therefore to find linear combinations of the $g_{j}$ that involve only partition monomials. For example, consider again the special case $\mu=(5,3,2,2)$ shown in Figure 4 . We will build a syzygy of $\left(g_{1}, \ldots, g_{7}\right)$; we begin by multiplying $g_{1}$ by $-y$ :

$$
-y \cdot g_{1}=-y \cdot\left(\begin{array}{l}
x^{4}-c_{3,0}^{4,0} \cdot x^{3}-c_{3,1}^{4,0} \cdot x^{3} y- \\
c_{2,0}^{4,0} \cdot x^{2}-c_{2,1}^{4,0} \cdot x^{2} y- \\
c_{1,0}^{4,0} \cdot x-c_{1,1}^{4,0} \cdot x y-c_{1,2}^{4,0} \cdot x y^{2}- \\
c_{0,0}^{4,0} \cdot 1-c_{0,1}^{4,0} \cdot y-c_{0,2}^{4,0} \cdot y^{2}-c_{0,3}^{4,0} \cdot y^{3}-c_{0,4}^{4,0} \cdot y^{4}
\end{array}\right)
$$

With reference to Figure 4, the multiplication by $-y$ shifts each term in $g_{1}$ one place to the right (and changes its sign). One easily lists the terms in (25) that are not scalar multiples of pattern monomials; we shall call such terms exposed terms, and their coefficient functions exposed coefficients:

$$
-x^{4} y, c_{3,1}^{4,0} \cdot x^{3} y^{2}, c_{2,1}^{4,0} \cdot x^{2} y^{2}, c_{1,2}^{4,0} \cdot x y^{3}, c_{0,4}^{4,0} \cdot y^{5} .
$$


Since these terms involve, respectively, the second, third, fourth, fifth, and seventh leading monomials, one sees easily that the dot product

$$
\left(-y, 1,-c_{3,1}^{4,0},-c_{2,1}^{4,0},-c_{1,2}^{4,0}, 0,-c_{0,4}^{4,0}\right) \cdot\left(g_{1}, g_{2}, \ldots, g_{7}\right)
$$

is an $\mathcal{O}_{U_{\mu}}$-linear combination of partition monomials. (Each exposed term in $-y \cdot g_{1}$ is cancelled by the addition of the appropriate multiple of the corresponding $g_{j}$.) Therefore, by Lemma 4.1.1, the tuple

$$
\mathrm{h}_{1}=\left(-y, 1,-c_{3,1}^{4,0},-c_{2,1}^{4,0},-c_{1,2}^{4,0}, 0,-c_{0,4}^{4,0}\right)
$$

is a syzygy of $\left(g_{1}, \ldots, g_{7}\right)$.

To build a second syzygy, we multiply the polynomial $g_{2}$, with leading term $x^{4} y$, by $-y$. In this case, one of the exposed terms, namely $-x^{4} y^{2}$, is not a scalar multiple of a leading monomial. To cancel this exposed term, we add $x \cdot g_{3}$ to the linear combination. Doing this introduces additional exposed terms, all of which are scalar multiples of leading monomials. Therefore, we can proceed as before to add appropriate multiples of the $g_{j}$ to cancel all the remaining exposed terms, and thereby obtain the following syzygy:

$$
\mathrm{h}_{2}=\left(c_{3,0}^{3,2},-y+c_{3,1}^{3,2}, x-c_{3,1}^{4,1},-c_{2,1}^{4,1}+c_{1,2}^{3,2},-c_{1,2}^{4,1}+c_{0,3}^{3,2}, c_{0,4}^{3,2},-c_{0,4}^{4,1}\right) .
$$

Continuing in this way, we construct six syzygies; these we gather together as the rows of the following matrix $\mathbf{m}_{(5,3,2,2)}$ :

(26)

$$
\left[\begin{array}{ccccccc}
-y & 1 & -c_{3,1}^{4,0} & -c_{2,1}^{4,0} & -c_{1,2}^{4,0} & 0 & -c_{0,4}^{4,0} \\
c_{3,0}^{3,2} & -y+c_{3,1}^{3,2} & x-c_{3,1}^{4,1} & -c_{2,1}^{4,1}+c_{1,2}^{3,2} & -c_{1,2}^{4,1}+c_{0,3}^{3,2} & c_{0,4}^{3,2} & -c_{0,4}^{4,1} \\
c_{3,2}^{2,2} & c_{3,1}^{2,2} & -1 & x+c_{1,2}^{2,2} & c_{0,3}^{2,2} & c_{0,4}^{2,2} & 0 \\
c_{3,0}^{1,3} & c_{3,1}^{1,3} & -c_{3,1}^{2,2} & -y-c_{2,1}^{2,2}+c_{1,2}^{1,3} & x-c_{1,2}^{2,2}+c_{0,3}^{1,3} & c_{0,4}^{1,3} & -c_{0,4}^{2,2} \\
0 & 0 & -c_{3,1}^{1,3} & -c_{2,1}^{1,3} & -y-c_{1,2}^{1,3} & 1 & -c_{0,4}^{1,3} \\
c_{3,0}^{0,5} & c_{3,1}^{0,5} & -c_{3,1}^{1,4} & -c_{2,1}^{1,4}+c_{1,2}^{0,5} & -c_{1,2}^{1,4}+c_{0,3}^{0,5} & -y+c_{0,4}^{0,5} & x-c_{0,4}^{1,4}
\end{array}\right] .
$$

We claim that the six syzygies $\mathrm{h}_{1}, \ldots, \mathrm{h}_{6}$ in (26) compose an $\mathcal{O}_{U_{\mu}}$-basis of the full syzygy module of $\left(g_{1}, \ldots, g_{7}\right)$; to prove this, and to prepare the ground for later sections, we turn to a more general treatment of the syzygy-making process.

4.2. $\mu$-Pseudosyzygies. Within the confines of this section and the next, we abuse our previous notation by using it in a more general context. Let $A$ be any commutative ring with identity (in place of $\mathcal{O}_{U_{\mu}}$ ), $B=A[x, y]$, and (as before) $\mathfrak{B}_{\mu}$ the set of partition monomials (4) associated to a partition $\mu$ of $n$. Let $g_{j} \in A[x, y], 1 \leq j \leq \Lambda_{\mu}$, be polynomials of the form (23), with the coefficients $c_{h k}^{r_{j}, s_{j}}$ arbitrarily chosen in $A$. We define a $\mu$-pseudosyzygy of $\left(g_{1}, \ldots, g_{\Lambda_{\mu}}\right)=\left(g_{k}\right)$ to be a $\left(\Lambda_{\mu}\right)$-tuple $\mathrm{f}=\left(f_{1}, \ldots, f_{\Lambda_{\mu}}\right)$ of elements of $B$ 
that satisfies

$$
\begin{aligned}
\left(g_{1}, \ldots, g_{\Lambda_{\mu}}\right) \cdot \mathrm{f} & =\sum_{k=1}^{\Lambda_{\mu}}\left(g_{k} \cdot f_{k}\right) \\
& =\left(\begin{array}{l}
\text { an } A \text {-linear combination of } \\
\text { partition monomials }
\end{array}\right) .
\end{aligned}
$$

We can construct $\mu$-pseudosyzygies of $\left(g_{k}\right)$ by repeating the process used in Section 4.1. More precisely, we execute:

Algorithm 4.2.1. Begin with $g_{1}$ and iterate through the $g_{k}, 1 \leq k<\Lambda_{\mu}$. At the $j$-th iteration, carry out the unique enumerated step that applies to $g_{j}$ to produce the $\mu$-pseudosyzygy $h_{j}$. In all, $\Lambda_{\mu}-1 \mu$-pseudosyzygies will be produced; note that the $h_{j}$ are indexed by the position of the $-y$ (for Types 1 and 2 ) or the -1 (for Type 3 ).

1) If $g_{j}$ is a top polynomial and $x^{r_{j}} y^{s_{j}+1}$ is a (top) leading monomial (equal to $x^{r_{j+1}} y^{s_{j+1}}$ ) multiply $g_{j}$ by $-y$, add $1 \cdot g_{j+1}$ to cancel $-x^{r_{j}} y^{s_{j}+1}$, and then add appropriate multiples of the $g_{k}$ to cancel the remaining exposed monomials, all of which are $A$-multiples of (side) leading monomials. We say that the the resulting $\mu$-pseudosyzygy is of Type $\mathbf{1}$; it has the value

$$
\mathrm{h}_{j}=\left(a_{j, 1}, a_{j, 2}, \ldots, a_{j, j-1},-y+a_{j, j}, 1, a_{j, j+2}, \ldots, a_{j, \Lambda_{\mu}}\right),
$$

where $a_{j, i} \in A$ is equal to $-c_{r_{i}, s_{i}-1}^{r_{j}, s_{j}}$ when $g_{i}$ is a side polynomial, and 0 otherwise.

2) If $g_{j}$ is a top polynomial and $x^{r_{j}} y^{s_{j}+1}$ is not a leading monomial, then $g_{j+1}$ has (side) leading monomial $x^{r_{j+1}} y^{s_{j+1}}=x^{r_{j}-1} y^{s_{j}+1}$. Multiply $g_{j}$ by $-y$, add $x \cdot g_{j+1}$ to cancel $-x^{r_{j}} y^{s_{j}+1}$, and then add appropriate multiples of the $g_{k}$ to cancel the remaining exposed terms, all of which are $A$-multiples of (top and side) leading monomials. We say that the resulting $\mu$-pseudosyzygy is of Type 2 ; it has the value

$$
\mathrm{h}_{j}=\left(a_{j, 1}, a_{j, 2}, \ldots, a_{j, j-1},-y+a_{j, j}, x+a_{j, j+1}, a_{j, j+2}, \ldots, a_{j, \Lambda_{\mu}}\right) \text {, }
$$

where $a_{j, i} \in A$ is equal to $\left(-c_{r_{i}, s_{i}-1}^{r_{j}, s_{j}}+c_{r_{i}-1, s_{i}}^{r_{j+1}, s_{j+1}}\right)$ when $g_{i}$ is both a top and a side polynomial, $-c_{r_{i}, s_{i}-1}^{r_{j}, s_{j}}$ when $g_{i}$ is only a side polynomial, and $c_{r_{i}-1, s_{i}}^{r_{j+1}, s_{j+1}}$ when $g_{i}$ is only a top polynomial.

3) If $g_{j}$ is a side polynomial and $x^{r_{j}-1} y^{s_{j}}$ is a (side) leading monomial (equal to $x^{r_{j+1}} y^{s_{j+1}}$ ), multiply $g_{j}$ by -1 , add $x \cdot g_{j+1}$ to cancel $x^{r_{j}} y^{s_{j}}$, and then add appropriate multiples of the $g_{k}$ to cancel the remaining exposed monomials, all of which are $A$-multiples of (top) leading monomials. We say that the resulting $\mu$-pseudosyzygy is of Type $\mathbf{3}$; it has the value

$$
\mathrm{h}_{j}=\left(a_{j, 1}, a_{j, 2}, \ldots, a_{j, j-1},-1, x+a_{j, j+1}, a_{j, j+2}, \ldots, a_{j, \Lambda_{\mu}}\right),
$$


where $a_{j, i} \in A$ is equal to $c_{r_{i}-1, s_{i}}^{r_{j+1}, s_{j+1}}$ when $g_{i}$ is a top polynomial, and 0 otherwise.

Remark 4.2.2. For future reference, we highlight that the $\mu$-pseudosyzygies $\mathrm{h}_{j}$ of Type 1 in Algorithm 4.2.1 have nonzero coefficients $a_{j, i}$ only in positions corresponding to side monomials; likewise, the $\mathrm{h}_{j}$ of Type 3 have nonzero coefficients $a_{j, i}$ only in positions corresponding to top monomials. The matrix of syzygies (26) provides an example.

4.3. Main theorem on $\mu$-Pseudosyzygies. We retain the notation of the previous section: $A$ is a commutative ring, $B=A[x, y], g_{j} \in B, 1 \leq j \leq$ $\Lambda_{\mu}$, are polynomials of the form (23), with the coefficients $c_{h k}^{r_{j}, s_{j}}$ arbitrarily chosen in $A$, and $\mathrm{h}_{1}, \ldots, \mathrm{h}_{\Lambda_{\mu}-1}$ are the $\mu$-pseudosyzygies of $\left(g_{j}\right)$ produced by Algorithm 4.2.1. We write $\mathrm{H} \subseteq B^{\Lambda_{\mu}}$ for the $B$-linear span of the $\mathrm{h}_{k}$. We shall soon show that every $\mathrm{H}$-coset has a unique representative $\mathrm{f}^{\prime}=\left(f_{1}^{\prime}, \ldots, f_{\Lambda_{\mu}}^{\prime}\right)$ with the following properties:

1) $f_{k}^{\prime}=0$ for all $k<\Lambda_{\mu}$ such that $g_{k}$ is not a top polynomial, and

2) each nonzero $f_{k}^{\prime}$ with $k<\Lambda_{\mu}$ is an element of $A[x]$.

We say that a $B^{\Lambda_{\mu}}$-tuple $\mathrm{f}^{\prime}$ with these properties is side-minimized, because the non-top entries $f_{k}^{\prime}$ for $k<\Lambda_{\mu}$ are all 0 , and because the other entries (except for the $\Lambda_{\mu}$-th), being elements of $A[x]$, cause only upward, not sideways, motion of monomials when they multiply their corresponding $g_{k}$ (see Figure 4).

Our main result on $\mu$-pseudosyzygies is Theorem 4.3.6; since the proof is lengthy, we break it up into a series of lemmas.

Lemma 4.3.1. A nontrivial B-linear combination of the $\mu$-pseudosyzygies $\mathrm{h}_{1}, \ldots, \mathrm{h}_{\Lambda_{\mu}-1}$ can never be side-minimized. In particular, the $\mathrm{h}_{k}$ form a $B$-linearly independent set.

Proof. Suppose given a nontrivial $B$-linear combination

$$
\gamma=\gamma_{1} \cdot h_{1}+\gamma_{2} \cdot h_{2}+\cdots+\gamma_{\Lambda_{\mu}-1} \cdot h_{\Lambda_{\mu}-1}
$$

that is side-minimized. Let $\gamma_{q}$ have maximal $y$-degree among the nonzero $\gamma_{k} \in B$. If $\mathrm{h}_{q}$ is of Type 1 or Type 2 (see Algorithm 4.2.1), so that the $q$-th component of $\mathrm{h}_{q}$ has the form $-y+a_{q, q}$, we have that the $q$-th component of $\gamma$ has the form

$$
\gamma_{q} \cdot\left(-y+a_{q, q}\right)+\sum_{k \neq q} \gamma_{k} \cdot\left(a_{k, q} \text { or } x+a_{k, q}\right), \quad a_{q, q}, a_{k, q} \in A .
$$

A moment's reflection shows that the terms of maximum $y$-degree in $-y \cdot \gamma_{q}$ cannot cancel out of this sum, which implies that the $q$-th component of $\gamma$ does not lie in $A[x]$; this yields a contradiction, since $q<\Lambda_{\mu}$, and $\gamma$ is by hypothesis side-minimized. From this we deduce that a nonzero coefficient $\gamma_{k}$ of maximal $y$-degree cannot multiply an $\mathrm{h}_{k}$ of Type 1 or 2 ; whence, $\mathrm{h}_{q}$ 
must be of Type 3 (see Algorithm 4.2.1), with $q$-th component equal to -1 . Therefore, the $q$-th component of $\gamma$ has the form

$$
\gamma_{q} \cdot(-1)+\gamma_{q-1} \cdot\left(x+a_{q-1, q}\right)+\sum_{k \neq q, q-1} \gamma_{k} \cdot a_{k, q}, \quad a_{q-1, q}, a_{k, q} \in A .
$$

Since $g_{q}$ is side polynomial that is not also a top polynomial, we have from Remark 4.2.2 that the only nonzero $a_{k, q}$ 's in (29) are those for which $\mathrm{h}_{k}$ is of Type 1 or 2 , which we recently saw implies that $\gamma_{k}$ has $y$-degree less than the (maximal) $y$-degree of $\gamma_{q}$. Therefore the terms of maximal $y$-degree in (29) can only cancel - as they must, since the $q$-th component of the sideminimized linear combination $\gamma$ is 0 - provided $\gamma_{q-1}$ has $y$-degree equal to the $y$-degree of $\gamma_{q}$, which again forces $\mathrm{h}_{q-1}$ to be of Type 3. Iterating this argument, we see that $h_{q-2}, h_{q-3}, \ldots$, must all be of Type 3 . Since $h_{1}$ is always of Type 1 or 2 , we eventually achieve a contradiction. It follows that the only side-minimized $B$-linear combination of the $\mathrm{h}_{k}$ is the trivial one. The $B$-linear independence of the $\mathrm{h}_{k}$ is an immediate consequence.

Remark 4.3.2. The basic idea of the preceding proof comes from $[\mathbf{9}$, proof of Proposition 4.1, page 51].

Lemma 4.3.3. Let $\mathrm{f}=\left(f_{1}, \ldots, f_{\Lambda_{\mu}}\right)$ be an arbitrary element of $B^{\Lambda_{\mu}}$. Then we can express $f$ uniquely in the form

$$
\mathrm{f}=\left(\sum_{k=1}^{\Lambda_{\mu}-1} b_{k} \cdot \mathrm{h}_{k}\right)+\mathrm{f}^{\prime}, b_{k} \in B,
$$

where $\mathrm{f}^{\prime} \in B^{\Lambda_{\mu}}$ is side-minimized. In brief, every $\mathrm{H}$-coset has a unique side-minimized representative.

Proof. We organize the operations needed to express $f$ in the desired form into a repeated alternation of two sub-procedures that we call column clearing and reduction of $y$-degree. In this context, a column refers to a maximal subset of the leading monomials having the same $y$-degree; for example, in Figure 4, we have six columns, five of which consist of one leading monomial, and one of which consists of two leading monomials (namely, $x^{2} y^{2}$ and $\left.x^{3} y^{2}\right)$.

Column clearing. Let $x^{r_{j}} y^{s_{j}}$ be a side leading monomial that is not of lowest $x$-degree in its column (for example, $x^{3} y^{2}$ in Figure 4). Then the syzygy $\mathrm{h}_{j}$ is of Type 3 (see Algorithm 4.2.1), with -1 in the $j$-th component, $x+a_{j, j+1}$ in the $j+1$ 'st component, and otherwise nonzero $a_{j, k}$ 's only in components associated to top polynomials $g_{k}$. Therefore we can write

$$
\begin{aligned}
\mathrm{f} & =-f_{j} \cdot \mathrm{h}_{j}+\left(\tilde{\mathrm{f}}+f_{j} \cdot \mathrm{h}_{j}\right) \\
& =-f_{j} \cdot \mathrm{h}_{j}+\widetilde{\mathrm{f}}
\end{aligned}
$$


in which the first summand lies in $\mathrm{H}$ and the second is a $\Lambda_{\mu}$-tuple that is 0 in the $j$-th component and otherwise differs from $\mathrm{f}$ only in components associated to top monomials and the $(j+1)$-st component. If $x^{r_{j}-1} y^{s_{j}}$ (the leading monomial immediately below $\left.x^{r_{j}} y^{s_{j}}\right)$ is not of lowest $x$-degree in the column, then we may apply this procedure to $\widetilde{f}=\left(\widetilde{f}_{1}, \ldots, \widetilde{f}_{\Lambda_{\mu}}\right)$ to obtain

$$
\begin{aligned}
\mathrm{f} & =\left(-f_{j} \cdot \mathrm{h}_{j}-\tilde{f}_{j+1} \cdot \mathrm{h}_{j+1}\right)+\left(\widetilde{\mathrm{f}}+\tilde{f}_{j+1} \cdot \mathrm{h}_{j+1}\right) \\
& =\left(-f_{j} \cdot \mathrm{h}_{j}-\widetilde{f}_{j+1} \cdot \mathrm{h}_{j+1}\right)+\widetilde{\widetilde{f}}
\end{aligned}
$$

in which $\widetilde{\widetilde{f}}$ is 0 in the $(j+1)$-st component, and otherwise differs from $\tilde{f}$ only in components associated to top monomials as well as the $(j+2)$-nd component (in particular, $\widetilde{\widetilde{f}}$ is still 0 in the $j$-th component). It is now clear that by working column-by-column and starting at the top of each column, we can write $f$ in the form

$$
\mathrm{f}=\left(\sum_{k=1}^{\Lambda_{\mu}-1} b_{k} \cdot \mathrm{h}_{k}\right)+\mathrm{f}_{\mathrm{cc}}, b_{k} \in B,
$$

where $\mathrm{f}_{\mathrm{cc}}$ is zero in all components except for those associated to top monomials and the last component (associated to the leading monomial $y^{p_{1}}$ ). At this point we have completed the column clearing operation on $\mathrm{f}$.

Remark 4.3.4. Following a column clearing operation on $f$, the maximum $y$-degree among the components of $\mathrm{f}_{\mathrm{cc}}$ is no greater than the maximum $y$ degree among the components of $f$. Indeed, the column clearing operation can only modify the components of $f$ in $y$-degrees $\leq$ the maximum $y$-degree among the $f_{j}$ corresponding to the positions that are set to zero by the process.

Reduction of $y$-degree. Let $\mathrm{f}=\left(f_{1}, \ldots, f_{\Lambda_{\mu}}\right) \in B^{\Lambda_{\mu}}$ and let $s$ be the maximal $y$-degree (achieved at $f_{j}$ ) among the components of $\mathrm{f}$ associated to top monomials. We write

$$
f_{j}=q_{s}(x) y^{s}+(\text { lower degree terms in } y)
$$

(we here view the elements of $B=A[x, y]$ as polynomials in $y$ with coefficients in $A[x]$ ). If $s>0$, we may use the syzygy $\mathrm{h}_{j}$, which is of Type 1 or 2 with $-y+a_{j, j}$ in the $j$-th component, to rewrite $\mathrm{f}$ as follows:

$$
\begin{aligned}
\mathrm{f} & =-q_{s}(x) y^{s-1} \cdot \mathrm{h}_{j}+\left(\mathfrak{f}+q_{s}(x) y^{s-1} \cdot \mathrm{h}_{j}\right) \\
& =-q_{s}(x) y^{s-1} \cdot \mathrm{h}_{j}+\widetilde{\mathrm{f}}
\end{aligned}
$$

in which the $y$-degree of the $j$-th component of $\widetilde{\mathrm{f}}$ is $\leq s-1$, and the other components of $\widetilde{f}$ differ from the corresponding components of $f$ only in $y$ degree $s-1$. If the maximal $y$-degree among the components of $\widetilde{f}$ associated 
to top monomials is still $s$, the process can be iterated to yield

$$
\mathrm{f}=\left(\sum_{k=1}^{\Lambda_{\mu}-1} b_{k} \cdot \mathrm{h}_{k}\right)+\mathrm{f}_{y \text {-red }}, b_{k} \in B,
$$

where $\mathrm{f}_{y \text {-red }}$ has $y$-degree $\leq s-1$ in all components associated to top monomials, and otherwise differs from $\mathrm{f}$ only in $y$-degree $s-1$. This completes the process of reduction of $y$-degree.

To complete the proof of the lemma, we begin with an arbitrary $f=$ $\left(f_{1}, \ldots, f_{\Lambda_{\mu}}\right)$, and apply column clearing to write $\mathrm{f}$ in the form (30). Let $s$ be the maximum $y$-degree among the components of $\mathrm{f}_{\mathrm{cc}}$ corresponding to top monomials. By applying reduction of $y$-degree to $\mathrm{f}_{\mathrm{cc}}$, we write $\mathrm{f}$ in the form (31), where $\mathrm{f}_{y \text {-red }}$ has $y$-degree $\leq s-1$ in each top position and in each position that is zeroed out by column clearing (because the latter positions are 0 in $\mathrm{f}_{\mathrm{cc}}$, and reduction of $y$-degree can change them only in $y$-degree $s-1$ ). In light of Remark 4.3.4, we see that column clearing applied to $\mathrm{f}_{y \text {-red }}$ will express $f$ again in the form (30), where now the maximum $y$-degree among the components of $\mathrm{f}_{\mathrm{cc}}$ corresponding to top monomials is $\leq s-1$. By iterating the alternation of reduction of $y$-degree and column clearing, we eventually obtain an expression for $f$ as specified in the statement of the lemma; that is, we produce a side-minimized representative $f^{\prime}$ for the coset $f+H$. Given a second such side-minimized representative $f^{\prime \prime}$, we have that $f^{\prime}-f^{\prime \prime}$ is a side-minimized element of $\mathrm{H}$; Lemma 4.3.1 now yields $\mathrm{f}^{\prime}-\mathrm{f}^{\prime \prime}=0$, which proves the uniqueness of the side-minimized representative and completes the proof of the lemma.

Lemma 4.3.5. If $\mathrm{f}=\left(f_{1}, \ldots, f_{\Lambda_{\mu}}\right)$ is a $\mu$-pseudosyzygy of $\left(g_{k}\right)$ that is sideminimized, then $\mathrm{f}=0$.

Proof. We first suppose that $f_{\Lambda_{\mu}} \neq 0$. Viewing $f_{\Lambda_{\mu}}$ as a polynomial in $y$ with coefficients in $A[x]$, we let $q(x) y^{s}$ be the term of maximal $y$-degree. Recall from (16) that we write $p_{1}$ for the largest part in the partition $\mu$; whence, the term of maximal $y$-degree in $f_{\Lambda_{\mu}} \cdot g_{\Lambda_{\mu}}$ is $q(x) y^{p_{1}+s}$ (as an example, see Figure 4 ). Since the other nonzero products $f_{k} \cdot g_{k}, 1 \leq k<\Lambda_{\mu}$, have maximal $y$-degree $<p_{1}\left(f_{k}\right.$ is either 0 or in $A[x]$, by hypothesis, and the maximal $y$-degree of every top $g_{k}$ is $<p_{1}$ ), we have that the term $q(x) y^{p_{1}+s}$ cannot cancel out of the expression $\sum_{k=1}^{\Lambda_{\mu}}\left(g_{k} \cdot f_{k}\right)$ in Equation (27), a contradiction. It follows that $f_{\Lambda_{\mu}}=0$.

Assume now that not all of the remaining components of $f$ are 0 . Among the nonzero $f_{k}, 1 \leq k<\Lambda_{\mu}$, let $f_{j}$ have maximal $x$-degree $d$, and let $a x^{d}$ be the corresponding term $(a \in A)$. Recall that the (top) leading monomial of $g_{j}$ is $x^{r_{j}} y^{s_{j}}$. The product $f_{j} \cdot g_{j}$ contributes the term $a x^{d+r_{j}} y^{s_{j}}$ to the expression $\sum_{k=1}^{\Lambda_{\mu}}\left(g_{k} \cdot f_{k}\right)$ in (27); if this term is to cancel out, as it 
must, then for at least one $j^{\prime} \neq j$ with $g_{j^{\prime}}$ a top polynomial, the product $f_{j^{\prime}} \cdot g_{j^{\prime}}$ must contain a term of the form $a^{\prime} x^{r_{j}} y^{s_{j}}$. But $f_{j^{\prime}}$ lies in $A[x]$, by hypothesis; moreover, the (top) leading monomial of $g_{j^{\prime}}$ must have $y$-degree $\neq s_{j}$, since distinct top monomials must differ in $y$-degree. Therefore, among the terms of $g_{j^{\prime}}$ having $y$-degree $s_{j}$, the largest possible $x$-degree is $r_{j}-1$. This means that the $x$-degree of $f_{j^{\prime}}$ must be at least $d+1$, which contradicts the maximality of $d$. We conclude that $f_{k}=0$ for all $k<\Lambda_{\mu}$, and the lemma is proved.

We are now ready to state and prove our main result on $\mu$-pseudosyzygies. Let $\mathfrak{G} \subseteq B=A[x, y]$ denote the ideal generated by the polynomials $g_{k}$. Observe that the proof of Lemma 3.2.3 carries over to show that $B / \mathfrak{G}$ is generated as an $A$-module by the partition monomials.

Theorem 4.3.6. If $B / \mathfrak{G}$ is free over $A$ with the partition monomials constituting a basis, then any $\mu$-pseudosyzygy of $\left(g_{k}\right)$ is in fact a syzygy of $\left(g_{k}\right)$; in this case, $\left\{\mathrm{h}_{1}, \ldots, \mathrm{h}_{\Lambda_{\mu}-1}\right\}$ is a B-basis of the first syzygy module of $\left(g_{k}\right)$. Conversely, if the $\mu$-pseudosyzygies $\mathrm{h}_{j}$ are all syzygies of $\left(g_{k}\right)$, then the $A$ module $B / \mathfrak{G}$ is free with basis given by the partition monomials.

Proof. First suppose that $B / \mathfrak{G}$ is $A$-free with basis consisting of the partition monomials, and let $\mathrm{f}=\left(f_{1}, \ldots, f_{\Lambda_{\mu}}\right)$ be a $\mu$-pseudosyzygy of the $\left(g_{k}\right)$. To prove that $f$ is a syzygy of $\left(g_{k}\right)$, we repeat the argument of Lemma 4.1.1: We have that

$$
L=\sum_{k=1}^{\Lambda_{\mu}}\left(f_{k} \cdot g_{k}\right) \equiv 0 \quad(\bmod \mathfrak{G})
$$

and

$$
L=\text { (a linear combination of partition monomials). }
$$

But by the freeness hypothesis, the only $A$-linear combination of partition monomials that is $\equiv 0(\bmod \mathfrak{G})$ is the trivial one; that is, $L=0$, which implies that $f$ is a syzygy of $\left(g_{k}\right)$.

In particular, the $\mu$-pseudosyzygies $\mathrm{h}_{1}, \ldots, \mathrm{h}_{\Lambda_{\mu}-1}$ given by Algorithm 4.2.1 are all syzygies of $\left(g_{k}\right)$; we must show that the $\mathrm{h}_{j}$ form a $B$-basis of the syzygy module. Since the $\mathrm{h}_{j}$ form a $B$-linearly independent set, by Lemma 4.3.1, it remains to show that any given syzygy $\mathrm{f}=\left(f_{1}, \ldots, f_{\Lambda_{\mu}}\right)$ lies in $\mathrm{H}$, the $B$-linear span of the $\mathrm{h}_{j}$. However, by Lemma 4.3.3, we know that the coset $\mathrm{f}+\mathrm{H}$ has a unique side-minimized representative $\mathrm{f}^{\prime}$, and it is clear that $\mathrm{f}^{\prime}$ is also a syzygy (and therefore a $\mu$-pseudosyzygy) of $\left(g_{k}\right)$. Lemma 4.3 .5 now yields $\mathrm{f}^{\prime}=0$; whence, $\mathrm{f} \in \mathrm{H}$, as desired.

We turn now to the last assertion of the theorem. Suppose that the $\mathrm{h}_{j}$ are all syzygies (not just $\mu$-pseudosyzygies) of $\left(g_{k}\right)$. As noted prior to the statement of the theorem, the $A$-module $B / \mathfrak{G}$ is generated by the partition 
monomials; it remains to prove that the latter are $A$-linearly independent $(\bmod \mathfrak{G})$.

To this end, we assume given an $A$-linear combination of partition monomials $L$ such that $L \equiv 0(\bmod \mathfrak{G})$. Since $L \in \mathfrak{G}$, there exists a $\mu$ pseudysyzygy $\mathrm{f}=\left(f_{1}, \ldots, f_{\Lambda_{\mu}}\right)$ of $\left(g_{k}\right)$ such that

$$
L=\mathrm{f} \cdot\left(g_{1}, \ldots, g_{\Lambda_{\mu}}\right)=\sum_{k=1}^{\Lambda_{\mu}}\left(f_{k} \cdot g_{k}\right) .
$$

Let $\mathrm{f}^{\prime}$ be the unique side-minimized representative of $\mathrm{f}+\mathrm{H}$ given by Lemma 4.3.3. Since the $\mathrm{h}_{j}$ are assumed to be syzygies of $\left(g_{k}\right)$, we see that

$$
L=\mathrm{f} \cdot\left(g_{k}\right)=\mathrm{f}^{\prime} \cdot\left(g_{k}\right) ;
$$

that is, $\mathrm{f}^{\prime}$ is a side-minimized $\mu$-pseudosyzygy of $\left(g_{k}\right)$, and is perforce 0 by Lemma 4.3.5. We conclude that $L=0$, which implies that the partition monomials are $A$-linearly independent $(\bmod \mathfrak{G})$. This completes the proof of the theorem.

4.4. Corollaries in the main case. We now return to the case of main interest, in which $A=\mathcal{O}_{U_{\mu}}, B=A[x, y]$, and the polynomials $g_{j} \in B$ are those given by (23). In light of Proposition 3.2.1 and Lemma 3.2.2, the preceding theorem specializes to yield:

Corollary 4.4.1. The $\mu$-pseudosyzygies $\mathrm{h}_{1}, \ldots, \mathrm{h}_{\Lambda_{\mu}-1}$ of $\left(g_{j}\right)$ given by Algorithm 4.2.1 are in fact syzygies that constitute an $\mathcal{O}_{U_{\mu}}[x, y]$-basis of the first syzygy module of $\left(g_{j}\right)$.

Before stating the remaining corollaries, we introduce some notation. We denote the $\left(\Lambda_{\mu}-1\right) \times \Lambda_{\mu}$ matrix with rows given by the syzygies $\mathrm{h}_{j}$ as follows:

$$
\mathbf{m}_{\mu}=\left(\mathrm{h}_{1}, \mathrm{~h}_{2}, \ldots, \mathrm{h}_{\Lambda \mu-1}\right) .
$$

We also define the maps

$$
\begin{array}{ll}
\alpha_{\mu}: B^{\Lambda_{\mu}-1} \rightarrow B^{\Lambda_{\mu}}, & \left(b_{1}, \ldots, b_{\Lambda_{\mu}-1}\right) \mapsto\left(b_{1}, \ldots, b_{\Lambda_{\mu}-1}\right) \cdot \mathbf{m}_{\mu}, \\
\beta_{\mu}: B^{\Lambda_{\mu}} \rightarrow B, & \left(b_{1}, \ldots, b_{\Lambda_{\mu}}\right) \mapsto \sum_{k=1}^{\Lambda_{\mu}} b_{k} \cdot g_{k} .
\end{array}
$$

Corollary 4.4.2. The composition

$$
0 \longrightarrow B^{\Lambda_{\mu}-1} \stackrel{\alpha_{\mu}}{\longrightarrow} B^{\Lambda_{\mu}} \stackrel{\beta_{\mu}}{\longrightarrow} B \longrightarrow B / \mathfrak{I}_{\mu} \longrightarrow 0
$$

is a free resolution of the $B$-module $B / \mathfrak{I}_{\mu}$ of length 2 .

Proof. The exactness of the sequence at $B$ follows from Proposition 3.2.1, which states that $\mathfrak{I}_{\mu}$ is generated by the $g_{j}, 1 \leq j \leq \Lambda_{\mu}$. Since

$$
\alpha_{\mu}\left(\left(b_{1}, \ldots, b_{\Lambda_{\mu}-1}\right)\right)=\sum_{k=1}^{\Lambda \mu-1}\left(b_{k} \cdot \mathrm{h}_{k}\right),
$$


the exactness at $B^{\Lambda_{\mu}}$ restates that the syzygyes $\mathrm{h}_{k}$ span the full syzygy module of $\left(g_{j}\right)$, which is the kernel of $\beta_{\mu}$. Finally, the injectivity of $\alpha_{\mu}$ is equivalent to the linear independence of the $h_{k}$.

Corollary 4.4.3. For any A-module $N$, one obtains an exact sequence by applying the functor $(\cdot) \otimes_{A} N$ to the free resolution of $B / \mathfrak{I}_{\mu}$ given in Corollary 4.4.2.

Proof. Since $B=A[x, y]$, we have that the free resolution of the preceding corollary is a free resolution of $B / \mathfrak{I}_{\mu}$ as an $A$-module. Therefore, the modules $\operatorname{Tor}_{q}^{A}\left(N, B / \mathfrak{I}_{\mu}\right)$ are the homology modules of the tensored sequence. But since $B / \mathfrak{I}_{\mu}$ is flat (in fact, free, by Lemma 3.2.2) over $A$, we have that

$$
\operatorname{Tor}_{q}^{A}\left(N, B / \mathfrak{I}_{\mu}\right) \approx \operatorname{Tor}_{q}^{A}\left(B / \mathfrak{I}_{\mu}, N\right)=0 ;
$$

whence, the corollary.

We end this section with:

Theorem 4.4.4. The generators $g_{j}$ of the ideal $\mathfrak{I}_{\mu}$ may be recovered (up to sign) as the maximal minors of the matrix $\mathbf{m}_{\mu}$ as follows:

$$
g_{j}=\operatorname{det}\left(e_{j}, \mathrm{~h}_{1}, \ldots, \mathrm{h}_{\Lambda_{\mu}-1}\right), \quad 1 \leq j \leq \Lambda_{\mu},
$$

where $e_{j}$ is the standard unit $\Lambda_{\mu}$-tuple with 1 in the $j$-th component.

Proof. Recall that $A$ is an integral domain. Since $B / \mathfrak{I}_{\mu}$ is finite and flat of degree $n$ over $A$, we have that $\mathfrak{I}_{\mu}$ is unmixed of codimension 2 ; we also have the free resolution of Corollary 4.4.2. In this situation, Schaps's proof of [11, Theorem 1, pp. 671-673] applies, and shows that if we put

$$
g_{j}^{\prime}=\operatorname{det}\left(e_{j}, \mathrm{~h}_{1}, \ldots, \mathrm{h}_{\Lambda_{\mu}-1}\right), 1 \leq j \leq \Lambda_{\mu},
$$

then there is a nonzero scalar $c \in \mathrm{k}$ such that $g_{j}^{\prime}=c \cdot g_{j}$ for all $j$. This result is a version of a theorem of Burch [2] (see also [3, Theorem 20.15, p. 502]), for which Schaps references [10, Ex. 8, p. 148]. It remains to show that $c=1$.

To this end, let $\lambda: A \rightarrow \mathrm{k}$ be the k-algebra map corresponding to the inclusion of the point $I_{\mu} \in U_{\mu}$, and $\widetilde{\lambda}: A[x, y] \rightarrow \mathrm{k}[x, y]$ the map induced by $\lambda$. Recall from Remark 3.1.4 that $I_{\mu}$ is the point of $U_{\mu}$ at which all of the functions $c_{h k}^{r_{j}, s_{j}} \in \mathfrak{c}_{\mu}$ vanish; whence,

$$
\widetilde{\lambda}\left(g_{j}\right)=\text { the leading monomial } x^{r_{j}} y^{s_{j}}, 1 \leq j \leq \Lambda_{\mu},
$$

and therefore, since $g_{j}^{\prime}=c \cdot g_{j}$,

$$
\widetilde{\lambda}\left(g_{j}^{\prime}\right)=\widetilde{\lambda}\left(c \cdot g_{j}\right)=c \cdot x^{r_{j}} y^{s_{j}}, 1 \leq j \leq \Lambda_{\mu} .
$$

On the other hand, applying $\tilde{\lambda}$ to both sides of (32), we obtain

$$
\widetilde{\lambda}\left(g_{j}^{\prime}\right)=\operatorname{det}\left(e_{j}, \widetilde{\lambda}\left(\mathrm{h}_{1}\right), \ldots, \widetilde{\lambda}\left(\mathrm{h}_{\Lambda_{\mu}-1}\right)\right), 1 \leq j \leq \Lambda_{\mu},
$$


where we apply $\widetilde{\lambda}$ componentwise to each row of the matrix on the righthand side. Referring (for example) to (26), and reading all the $c_{h k}^{r_{j}, s_{j}}$ therein as 0 , we see that

$$
\widetilde{\lambda}\left(g_{1}^{\prime}\right)=\operatorname{det}\left(e_{1}, \widetilde{\lambda}\left(\mathrm{h}_{1}\right), \ldots, \widetilde{\lambda}\left(\mathrm{h}_{\Lambda_{\mu}-1}\right)\right)
$$

is the determinant of a lower-triangular matrix whose main diagonal comprises only 1's and $x$ 's. Moreover, there is one $x$ for each side monomial associated to $\mu$, and since the number of side monomials is equal to the number of parts $\ell$ of $\mu$, we may expand the determinant in (34) to obtain

$$
\widetilde{\lambda}\left(g_{1}^{\prime}\right)=x^{\ell}=x^{r_{1}} y^{s_{1}}
$$

(recall Remark 3.1.1). Confronting this with (33), we find that $c=1$, as desired.

\section{An explicit representation of $\mathcal{O}_{U_{\mu}}$ as a quotient ring.}

According to Proposition 3.1.2, the set $\mathfrak{c}_{\mu}$ (19) generates the affine coordinate ring $\mathcal{O}_{U_{\mu}}$ as a k-algebra. To represent this ring as a quotient, we introduce the set of indeterminates

$$
\begin{aligned}
\mathfrak{C}_{\mu} & =\left\{C_{h k}^{r_{j}, s_{j}} \mid c_{h k}^{r_{j}, s_{j}} \in \mathfrak{c}_{\mu}\right\} \\
& =\left\{C_{h k}^{r_{j}, s_{j}} \mid 1 \leq j \leq \Lambda_{\mu},(h, k) \in \mu\right\},
\end{aligned}
$$

and define the surjection

$$
\begin{gathered}
u_{\mu}^{*}: \mathrm{k}\left[\mathfrak{C}_{\mu}\right] \rightarrow \mathcal{O}_{U_{\mu}}, C_{h k}^{r_{j}, s_{j}} \mapsto c_{h k}^{r_{j}, s_{j}}, \text { which is the comorphism } \\
\text { of a closed immersion } u_{\mu}: U_{\mu} \rightarrow \operatorname{Spec}\left(\mathrm{k}\left[\mathfrak{C}_{\mu}\right]\right) .
\end{gathered}
$$

Our main goal in this section is to describe the kernel $\mathfrak{R}_{\mu}$ of $u_{\mu}^{*}$. We show that explicit generators of $\Re_{\mu}$ can be obtained from the $\mu$-pseudosyzygies studied in Sections 4.2 and 4.3. As an example, we compute these generators in case $\mu=(2,1)$, for which the associated monomial ideal is the "fat point" ideal

$$
I_{\mu}=\left(x^{2}, x y, y^{2}\right)=(x, y)^{2} \subseteq \mathrm{k}[x, y] .
$$

5.1. Generators of the kernel of $u_{\mu}^{*}$. We begin by defining the polynomials

$$
G_{j}=x^{r_{j}} y^{s_{j}}-\sum_{(h, k) \in \mu} C_{h k}^{r_{j}, s_{j}} x^{h} y^{k} \in \mathrm{k}\left[\mathfrak{C}_{\mu}\right][x, y], \quad 1 \leq j \leq \Lambda_{\mu} ;
$$

it is clear that each $G_{j}$ is a preimage of $g_{j}(23)$ under the map

$$
\widetilde{u}_{\mu}^{*}: \mathrm{k}\left[\mathfrak{C}_{\mu}\right][x, y] \rightarrow \mathcal{O}_{U_{\mu}}[x, y]
$$

induced by $u_{\mu}^{*}$ (36). We next execute Algorithm 4.2.1, with $A=\mathrm{k}\left[\mathfrak{C}_{\mu}\right]$ and $B=A[x, y]$, to generate the $\mu$-pseudosyzygies $\mathrm{H}_{1}, \ldots, \mathrm{H}_{\Lambda_{\mu}-1}$ of $\left(G_{k}\right)$. In 
particular, for $1 \leq j \leq \Lambda_{\mu}-1$, we have that

$$
\left(G_{1}, \ldots, G_{\Lambda_{\mu}}\right) \cdot \mathrm{H}_{j}=\sum_{(h, k) \in \mu} \rho_{h k}^{j} x^{h} y^{k}
$$

is an $A$-linear combination of partition monomials. Applying $\widetilde{u}_{\mu}^{*}$ componentwise to the $\left(\Lambda_{\mu}\right)$-tuples in (38), we see that $\widetilde{u}_{\mu}^{*}\left(\mathrm{H}_{j}\right)=\mathrm{h}_{j}$ is the $j$-th $\mu$-pseudosyzygy of $\left(g_{k}\right)$ generated by Algorithm 4.2.1, which is in fact a syzygy of $\left(g_{k}\right)$ by Corollary 4.4.1. It follows that

$$
\widetilde{u}_{\mu}^{*}\left(\sum_{(h, k) \in \mu} \rho_{h k}^{j} x^{h} y^{k}\right)=0 \in \mathcal{O}_{U_{\mu}}[x, y] ;
$$

whence,

$$
u_{\mu}^{*}\left(\rho_{h k}^{j}\right)=0 \in \mathcal{O}_{U_{\mu}} .
$$

In other words, the ideal

$$
\mathfrak{R}^{\prime}=\left(\left\{\rho_{h k}^{j} \mid 1 \leq j \leq \Lambda_{\mu}-1,(h, k) \in \mu\right\}\right) \subseteq \mathfrak{R}_{\mu} .
$$

Theorem 5.1.1. The functions $\rho_{h k}^{j} \in \mathrm{k}\left[\mathfrak{C}_{\mu}\right]$ generate the kernel of the surjection $u_{\mu}^{*}: \mathrm{k}\left[\mathfrak{C}_{\mu}\right] \rightarrow \mathcal{O}_{U_{\mu}} ;$ that is, $\mathfrak{R}^{\prime}=\mathfrak{R}_{\mu}$.

Proof. We write

$$
V=\operatorname{Spec}\left(\mathrm{k}\left[\mathfrak{C}_{\mu}\right] / \mathfrak{R}^{\prime}\right), \quad \mathcal{O}_{V}=\mathrm{k}\left[\mathfrak{C}_{\mu}\right] / \mathfrak{R}^{\prime},
$$

and observe that, by (39), the map (36) induces a surjection

$$
v^{*}: \mathcal{O}_{V} \rightarrow \mathcal{O}_{U_{\mu}}
$$

that is the comorphism of a closed immersion

$$
v: U_{\mu} \rightarrow V \text {. }
$$

Since the desired conclusion is equivalent to these maps being isomorphisms, we seek an inverse map

$$
\omega: V \rightarrow U_{\mu}
$$

By the universal property of $U_{\mu}$ given in Proposition 2.3.2, $\omega$ corresponds to a closed subscheme $Z \subseteq V \times \mathrm{A}_{\mathrm{k}}^{2}$ that is finite and flat of degree $n$ over $V$, and has fibers over k-points $v \in V$ that are cut out by ideals $I_{v} \in U_{\mu}$; we therefore seek such a family of subschemes.

Let

$$
u^{\prime}: \mathrm{k}\left[\mathfrak{C}_{\mu}\right] \rightarrow \mathcal{O}_{V}, \quad \widetilde{u}^{\prime}: \mathrm{k}\left[\mathfrak{C}_{\mu}\right][x, y] \rightarrow \mathcal{O}_{V}[x, y]
$$

denote, respectively, the quotient map and the map induced thereby on polynomials in $x$ and $y$, and set

$$
\begin{gathered}
g_{j}^{\prime}=\widetilde{u}^{\prime}\left(G_{j}\right), \quad 1 \leq j \leq \Lambda_{\mu}, \\
\mathfrak{G}^{\prime}=\left(g_{1}^{\prime}, \ldots, g_{\Lambda_{\mu}}^{\prime}\right) \subseteq \mathcal{O}_{V}[x, y] .
\end{gathered}
$$


Applying $\widetilde{u}^{\prime}$ to both sides of Equation (38) (componentwise to the $\left(\Lambda_{\mu}\right)$ tuples), and recalling that all the $\rho_{h k}^{j}$ vanish under $u^{\prime}$, we see that each $\mathrm{h}_{j}^{\prime}=$ $\widetilde{u}^{\prime}\left(\mathrm{H}_{j}\right)$, the $j$-th $\mu$-pseudosyzygy of $\left(g_{k}^{\prime}\right)$ produced by Algorithm 4.2.1, is in fact a syzygy of $\left(g_{k}^{\prime}\right)$. It now follows from the last statement of Theorem 4.3.6 that $\mathcal{O}_{V}[x, y] / \mathfrak{G}^{\prime}$ is free of dimension $n$ as an $\mathcal{O}_{V}$-module, with the partition monomials constituting a basis. Therefore, the map

$$
\operatorname{Spec}\left(\mathcal{O}_{V}[x, y] / \mathfrak{G}^{\prime}\right) \stackrel{F}{\longrightarrow} \operatorname{Spec}\left(\mathcal{O}_{V}\right)=V,
$$

induced by the natural map $\mathcal{O}_{V} \rightarrow \mathcal{O}_{V}[x, y] / \mathfrak{G}^{\prime}$, is finite and flat of degree $n$, and has fibers over k-points cut out by ideals $I \in U_{\mu}$. We define $\omega: V \rightarrow U_{\mu}$ to be the map corresponding to the family of subschemes $F$.

We claim that the composition of comorphisms

$$
\mathcal{O}_{V} \stackrel{v^{*}}{\longrightarrow} \mathcal{O}_{U_{\mu}} \stackrel{\omega^{*}}{\longrightarrow} \mathcal{O}_{V}
$$

is the identity map. This being granted, the theorem follows at once, since $v^{*}$, already known to be surjective, is now seen to be injective as well, and hence an isomorphism, as desired. It remains to prove the claim.

By definition, we have that the map $v^{*}$ behaves as follows on the images of the $C_{h k}^{r_{j}, s_{j}}$ :

$$
u^{\prime}\left(C_{h k}^{r_{j}, s_{j}}\right) \stackrel{v^{*}}{\mapsto} u_{\mu}^{*}\left(C_{h k}^{r_{j}, s_{j}}\right)=c_{h k}^{r_{j}, s_{j}} .
$$

Furthermore, from the universal property of $U_{\mu}$ (Proposition 2.3.2), the ideal $\mathfrak{G}^{\prime} \subseteq \mathcal{O}_{V}[x, y]$ that cuts out the family of subschemes inducing the map $\omega$ is the extension of the ideal $\left(g_{1}, \ldots, g_{\Lambda_{\mu}}\right)=\mathfrak{I}_{\mu} \subseteq \mathcal{O}_{U_{\mu}}[x, y]$ that cuts out the universal closed subscheme over $U_{\mu}$ (Proposition 3.2.1), under the map

$$
\widetilde{\omega}^{*}: \mathcal{O}_{U_{\mu}}[x, y] \rightarrow \mathcal{O}_{V}[x, y]
$$

induced by $\omega^{*}$. In other words, the polynomials

$$
\widetilde{\omega}^{*}\left(g_{j}\right)=x^{r_{j}} y^{s_{j}}-\sum_{(h, k) \in \mu} \omega^{*}\left(c_{h k}^{r_{j}, s_{j}}\right) x^{h} y^{k} \in \mathcal{O}_{V}[x, y], \quad 1 \leq j \leq \Lambda_{\mu},
$$

generate the ideal $\mathfrak{G}^{\prime}$. By construction, however, $\mathfrak{G}^{\prime}$ is generated by the polynomials

$$
g_{j}^{\prime}=\widetilde{u}^{\prime}\left(G_{j}\right)=x^{r_{j}} y^{s_{j}}-\sum_{(h, k) \in \mu} u^{\prime}\left(C_{h k}^{r_{j}, s_{j}}\right) x^{h} y^{k} \in \mathcal{O}_{V}[x, y], \quad 1 \leq j \leq \Lambda_{\mu} ;
$$

moreover, since the partition monomials give an $\mathcal{O}_{V^{-}}$basis of the quotient $\mathcal{O}_{V}[x, y] / \mathfrak{G}^{\prime}$, we have that $\mathfrak{G}^{\prime}$ contains a unique polynomial of the form

$$
\text { (leading term } \left.x^{r_{j}} y^{s_{j}}\right)+\left(\begin{array}{l}
\text { an } \mathcal{O}_{V} \text {-linear combination of } \\
\text { partition monomials }
\end{array}\right)
$$

for each $j$. From this it follows at once that

$$
c_{h k}^{r_{j}, s_{j}} \stackrel{\omega^{*}}{\mapsto} u^{\prime}\left(C_{h k}^{r_{j}, s_{j}}\right),
$$


which, in light of (41), shows that the composition (40) maps each element $u^{\prime}\left(C_{h, k}^{r_{j}, s_{j}}\right)$ to itself; since these elements generate the source as a k-algebra, we conclude that the composition is the identity, as claimed. This completes the proof of the theorem.

5.2. An example: $\mu=(\mathbf{2 , 1})$. To illustrate Theorem 5.1.1, we compute the $\mathrm{H}_{j}$ and the $\rho_{h k}^{j}$ in case $\mu=(2,1)$; the monomial ideal associated to $\mu$ is $\left(x^{2}, x y, y^{2}\right)=(x, y)^{2}$ (see Figure 5 ). Our set $\mathfrak{C}_{\mu=(2,1)}$ of indeterminates (35)

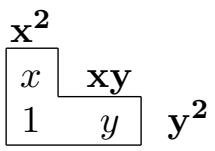

Figure 5. Diagram of $\mu=(2,1)$ with the partition monomials boxed in and the leading monomials shown in boldface.

consists of the nine coefficients $C_{h k}^{r_{j}, s_{j}}$ in the polynomials

$$
\begin{aligned}
& G_{0}=x^{2}-C_{0,0}^{2,0}-C_{1,0}^{2,0} x-C_{0,1}^{2,0} y, \\
& G_{1}=x y-C_{0,0}^{1,1}-C_{1,0}^{1,1} x-C_{0,1}^{1,1} y, \\
& G_{2}=y^{2}-C_{0,0}^{0,2}-C_{1,0}^{0,2} x-C_{0,1}^{0,2} y .
\end{aligned}
$$

It is easy to check that Algorithm 4.2.1 yields the following two pseudosyzygies:

$$
\begin{aligned}
& \mathrm{H}_{1}=\left(-y+C_{1,0}^{1,1}, x-C_{1,0}^{2,0}+C_{0,1}^{1,1},-C_{0,1}^{2,0}\right), \\
& \mathrm{H}_{2}=\left(C_{1,0}^{0,2},-y-C_{1,0}^{1,1}+C_{0,1}^{0,2}, x-C_{0,1}^{1,1}\right) .
\end{aligned}
$$

Computing the dot product (38) for $j=1$ and 2 , and reading off the coefficients $\rho_{h k}^{j}$ on the right-hand side, we obtain our set of generators for $\mathfrak{R}_{\mu=(2,1)}$ :

$$
\begin{aligned}
\rho_{0,0}^{1} & =-C_{1,0}^{1,1} C_{0,0}^{2,0}+C_{0,0}^{0,2} C_{0,1}^{2,0}+C_{0,0}^{1,1}\left(-C_{0,1}^{1,1}+C_{1,0}^{2,0}\right), \\
\rho_{1,0}^{1} & =-C_{0,0}^{1,1}-C_{0,1}^{1,1} C_{1,0}^{1,1}+C_{1,0}^{0,2} C_{0,1}^{2,0} \\
\rho_{0,1}^{1} & =-\left(C_{0,1}^{1,1}\right)^{2}+C_{0,0}^{2,0}+\left(C_{0,1}^{0,2}-C_{1,0}^{1,1}\right) C_{0,1}^{2,0}+C_{0,1}^{1,1} C_{1,0}^{2,0} \\
\rho_{0,0}^{2} & =-C_{0,1}^{0,2} C_{0,0}^{1,1}+C_{0,0}^{0,2} C_{0,1}^{1,1}+C_{0,0}^{1,1} C_{1,0}^{1,1}-C_{1,0}^{0,2} C_{0,0}^{2,0}, \\
\rho_{1,0}^{2} & =-C_{0,0}^{0,2}+C_{1,0}^{0,2} C_{0,1}^{1,1}-C_{0,1}^{0,2} C_{1,0}^{1,1}+\left(C_{1,0}^{1,1}\right)^{2}-C_{1,0}^{0,2} C_{1,0}^{2,0}, \\
\rho_{0,1}^{2} & =C_{0,0}^{1,1}+C_{0,1}^{1,1} C_{1,0}^{1,1}-C_{1,0}^{0,2} C_{0,1}^{2,0} .
\end{aligned}
$$

There is considerable redundancy among these generators; indeed, we have that

$$
\mathfrak{R}_{(2,1)}=\left(\rho_{1,0}^{1}, \rho_{0,1}^{1}, \rho_{1,0}^{2}\right),
$$


which follows from the relations

$$
\begin{aligned}
& \rho_{0,0}^{1}=\left(C_{0,1}^{1,1}-C_{1,0}^{2,0}\right) \rho_{1,0}^{1}-C_{1,0}^{1,1} \rho_{0,1}^{1}-C_{0,1}^{2,0} \rho_{1,0}^{2}, \\
& \rho_{0,0}^{2}=\left(C_{0,1}^{0,2}-C_{1,0}^{1,1}\right) \rho_{1,0}^{1}-C_{1,0}^{0,2} \rho_{0,1}^{1}-C_{0,1}^{1,1} \rho_{1,0}^{2}, \\
& \rho_{0,1}^{2}=-\rho_{1,0}^{1} .
\end{aligned}
$$

(We derive these relations in the next section.) Note that the generators in (44) express $C_{0,0}^{2,0}, C_{0,0}^{1,1}$, and $C_{0,0}^{0,2}$ as polynomials in the remaining $C^{\prime}$ 's (mod $\left.\mathfrak{R}_{(2,1)}\right)$; it follows from this that

$$
\mathcal{O}_{U_{(2,1)}} \approx \mathrm{k}\left[\mathfrak{C}_{(2,1)}\right] / \mathfrak{R}_{(2,1)} \approx \mathrm{k}\left[C_{1,0}^{2,0}, C_{0,1}^{2,0}, C_{1,0}^{1,1}, C_{0,1}^{1,1}, C_{1,0}^{0,2}, C_{0,1}^{0,2}\right] .
$$

In other words,

$$
\begin{aligned}
& U_{(2,1)} \text { is a six-dimensional affine cell in } \mathbf{H}^{3} \text { : that is, } U_{(2,1)} \text { is } \\
& \text { an open neighborhood in } \mathbf{H}^{3} \text { that is isomorphic to } \mathrm{A}_{\mathbf{k}}^{6} \text {. }
\end{aligned}
$$

We reconfirm this fact in Section 7 (second of Remarks 7.3.3) as one example of a more general sufficient condition for $U_{\mu}$ to be an affine cell (Corollary 7.3.2). In particular, this condition holds for all $\mu$ whose associated monomial ideal $I_{\mu}$ is a "fat point" ideal $(x, y)^{r}, r=1,2,3, \ldots$. The proof relies on a second set of generators of the ideal $\mathfrak{R}_{\mu}$ that we present in the next section.

\section{A second set of generators of the ideal $\mathfrak{R}_{\mu}$.}

In this section we present a second set of generators of the ideal $\mathfrak{R}_{\mu} \subseteq \mathrm{k}\left[\mathfrak{C}_{\mu}\right]$ that is more convenient for certain purposes than the set of generators given by Theorem 5.1.1. The key ingredient is provided by Theorem 4.4.4, which states that we can recover the polynomials $g_{k} \in \mathcal{O}_{U_{\mu}}[x, y]$ (up to sign) as the maximal minors of the matrix $\mathbf{m}_{\mu}$ whose rows are the basic syzygies $\mathrm{h}_{j}$ of $\left(g_{k}\right)$. As an example, we continue our study of the case $\mu=(2,1)$ that we began in Section 5.2.

6.1. The second set of generators. Retaining all previous notation, we begin by defining the $\left(\left(\Lambda_{\mu}-1\right) \times \Lambda_{\mu}\right)$-matrix

$$
\mathbf{M}_{\mu}=\left(\mathrm{H}_{1}, \mathrm{H}_{2}, \ldots, \mathrm{H}_{\Lambda_{\mu}-1}\right),
$$

with rows $\mathrm{H}_{j}$ the $\mu$-pseudosyzygies of $\left(G_{k}\right)$ introduced in Section 5.1. By analogy with the determinantal expression for $g_{j}$ given by Theorem 4.4.4, we define the polynomials

$$
\mathcal{D}_{j}=\operatorname{det}\left(e_{j}, \mathrm{H}_{1}, \ldots, \mathrm{H}_{\Lambda_{\mu}-1}\right) \in \mathrm{k}\left[\mathfrak{C}_{\mu}\right][x, y], \quad 1 \leq j \leq \Lambda_{\mu},
$$

where $e_{j}$ is the $j$-th standard unit vector. We noted in the discussion preceding Theorem 5.1.1 that the map $\widetilde{u}_{\mu}^{*}(37)$ maps each $G_{k}$ to $g_{k}$ and (acting 
componentwise) each $\mathrm{H}_{k}$ to $\mathrm{h}_{k}$ (and therefore $\mathbf{M}_{\mu}$ to $\mathbf{m}_{\mu}$ ). It follows from Theorem 4.4.4 that

$$
\widetilde{u}_{\mu}^{*}\left(\mathcal{D}_{j}\right)=g_{j}, \quad 1 \leq j \leq \Lambda_{\mu} ;
$$

since $g_{j}$ has the form (23), we are led to write each $\mathcal{D}_{j}$ in the form

$$
\mathcal{D}_{j}=x^{r_{j}} y^{s_{j}}-\sum_{(h, k) \in \mu} D_{h k}^{r_{j}, s_{j}} x^{h} y^{k}+\mathcal{N}_{j},
$$

where $D_{h k}^{r_{j}, s_{j}} \in \mathrm{k}\left[\mathfrak{C}_{\mu}\right]$ and $\mathcal{N}_{j} \in \operatorname{ker}\left(\widetilde{u}_{\mu}^{*}\right)$ involves only non-partition monomials (in $x$ and $y$ ), possibly including the leading monomials $x^{r_{k}} y^{s_{k}}$. Since $G_{j}$ and $\mathcal{D}_{j}$ both map to $g_{j}$ under $\widetilde{u}_{\mu}^{*}$, we have that $C_{h, k}^{r_{j}, s_{j}}$ and $D_{h, k}^{r_{j}, s_{j}}$ both map to $c_{h k}^{r_{j}, s_{j}}$ under $u_{\mu}^{*}$; whence,

$$
u_{\mu}^{*}\left(C_{h k}^{r_{j}, s_{j}}-D_{h k}^{r_{j}, s_{j}}\right)=0, \quad 1 \leq j \leq \Lambda_{\mu},(h, k) \in \mu .
$$

We set

$$
\delta_{h k}^{r_{j}, s_{j}}=C_{h k}^{r_{j}, s_{j}}-D_{h k}^{r_{j}, s_{j}}=C_{h k}^{r_{j}, s_{j}}+\left(\begin{array}{c}
\text { the coefficient of } x^{h} y^{k} \\
\text { in the polynomial } \mathcal{D}_{j}
\end{array}\right),
$$

and define the ideal

$$
\mathfrak{R}^{\prime \prime}=\left(\left\{\delta_{h k}^{r_{j}, s_{j}} \mid 1 \leq j \leq \Lambda_{\mu},(h, k) \in \mu\right\}\right) \subseteq \mathrm{k}\left[\mathfrak{C}_{\mu}\right] .
$$

Theorem 6.1.1. The functions $\delta_{h k}^{r_{j}, s_{j}} \in \mathrm{k}\left[\mathfrak{C}_{\mu}\right]$ generate the kernel of the surjection $u_{\mu}^{*}: \mathrm{k}\left[\mathfrak{C}_{\mu}\right] \rightarrow \mathcal{O}_{U_{\mu}} ;$ that is, $\mathfrak{R}^{\prime \prime}=\mathfrak{R}_{\mu}$.

We need the following:

Lemma 6.1.2. The $\mathrm{H}_{q}$ are syzygies of $\left(\mathcal{D}_{k}\right)$, that is,

$$
\mathrm{H}_{q} \cdot\left(\mathcal{D}_{1}, \ldots, \mathcal{D}_{\Lambda_{\mu}}\right)=0, \quad 1 \leq q \leq \Lambda_{\mu}-1 \text {. }
$$

Proof. If one replaces the first row $e_{j}$ of the determinant in (47) with $\mathrm{H}_{q}$, then the result must be 0 , since the matrix now has two equal rows. On the other hand, by linearity of the determinant in the first row, the value of this determinant is given by $\mathrm{H}_{q} \cdot\left(\mathcal{D}_{1}, \ldots, \mathcal{D}_{\Lambda_{\mu}}\right)$; whence, the lemma.

Proof of Theorem 6.1.1. By (49), we have that $\mathfrak{R}^{\prime \prime} \subseteq \mathfrak{R}_{\mu}$; it remains to establish the reverse inclusion. Recall from (38) that, for $1 \leq q \leq \Lambda_{\mu}-1$,

$$
\mathrm{H}_{q} \cdot\left(G_{1}, \ldots, G_{\Lambda_{\mu}}\right)=\sum_{(h, k) \in \mu} \rho_{h k}^{q} x^{h} y^{k} ;
$$

since $\mathrm{H}_{q}$ is a syzygy of $\left(\mathcal{D}_{k}\right)$, by the lemma, we may write

$$
\mathrm{H}_{q} \cdot\left(G_{1}-\mathcal{D}_{1}, \ldots, G_{\Lambda_{\mu}}-\mathcal{D}_{\Lambda_{\mu}}\right)=\sum_{(h, k) \in \mu} \rho_{h k}^{q} x^{h} y^{k} .
$$


By (48) and (50), we have that

$$
G_{j}-\mathcal{D}_{j}=\left(\sum_{(h, k) \in \mu}-\delta_{h k}^{r_{j}, s_{j}} x^{h} y^{k}\right)-\mathcal{N}_{j} .
$$

Now, since the term $\rho_{h k}^{q} x^{h} y^{k}$ on the right-hand side of (51) can involve only those terms of the factors on the left-hand side of the form

$$
a x^{r} y^{s}, \text { with } r \leq h, s \leq k, \text { so that }(r, s) \in \mu,
$$

we see that (51) expresses $\rho_{h k}^{q}$ as a $\mathrm{k}\left[\mathfrak{C}_{\mu}\right]$-linear combination of the $\left\{\delta_{h k}^{r_{j}, s_{j}}\right\}$. Since this holds for all $1 \leq q \leq \Lambda_{\mu}-1,(h, k) \in \mu$, we may invoke Theorem 5.1.1 to conclude that

$$
\mathfrak{R}_{\mu}=\left(\left\{\rho_{h k}^{q}\right\}\right) \subseteq \mathfrak{R}^{\prime \prime}
$$

which completes the proof.

6.2. An example continued: $\mu=(2,1)$. For an example, we return to the case $\mu=(2,1)$ considered in Section 5.2. Recalling (42), we see that

$$
\begin{aligned}
& \mathcal{D}_{1}=\operatorname{det}\left[\begin{array}{ccc}
1 & 0 & 0 \\
-y+C_{1,0}^{1,1} & x-C_{1,0}^{2,0}+C_{0,1}^{1,1} & -C_{0,1}^{2,0} \\
C_{1,0}^{0,2} & -y-C_{1,0}^{1,1}+C_{0,1}^{0,2} & x-C_{0,1}^{1,1}
\end{array}\right] \text {, } \\
& \mathcal{D}_{2}=\operatorname{det}\left[\begin{array}{ccc}
0 & 1 & 0 \\
-y+C_{1,0}^{1,1} & x-C_{1,0}^{2,0}+C_{0,1}^{1,1} & -C_{0,1}^{2,0} \\
C_{1,0}^{0,2} & -y-C_{1,0}^{1,1}+C_{0,1}^{0,2} & x-C_{0,1}^{1,1}
\end{array}\right] \text {, } \\
& \mathcal{D}_{3}=\operatorname{det}\left[\begin{array}{ccc}
0 & 0 & 1 \\
-y+C_{1,0}^{1,1} & x-C_{1,0}^{2,0}+C_{0,1}^{1,1} & -C_{0,1}^{2,0} \\
C_{1,0}^{0,2} & -y-C_{1,0}^{1,1}+C_{0,1}^{0,2} & x-C_{0,1}^{1,1}
\end{array}\right] \text {; }
\end{aligned}
$$

whence,

$$
\begin{array}{cc}
\mathcal{D}_{1}= & x^{2}-C_{1,0}^{2,0} x-C_{0,1}^{2,0} y-\left(C_{0,1}^{1,1}\right)^{2}+C_{0,1}^{0,2} C_{0,1}^{2,0} \\
-C_{1,0}^{1,1} C_{0,1}^{2,0}+C_{0,1}^{1,1} C_{1,0}^{2,0}
\end{array}
$$

Remark 6.2.1. Note that the summands $\mathcal{N}_{j}$ (48) of the $\mathcal{D}_{j}$ in (53) all vanish. This rather special phenomenon has some interesting consequences that, being tangential to our present purposes, will not be discussed in this paper. (For example, when the $\mathcal{N}_{j}$ all vanish, it can be shown that the pseudosyzygies $\mathrm{H}_{k}$ are invariant under the substitutions $C_{h k}^{r_{i}, s_{i}} \mapsto D_{h k}^{r_{i}, s_{i}}$.) 
We will, however, "explain" why the phenomenon occurs in this and similar cases in the next section (Corollary 7.2.2).

Computing the functions $\delta_{h k}^{r_{j}, s_{j}}(50)$, we find that

$$
\begin{aligned}
\delta_{0,0}^{2,0} & =C_{0,0}^{2,0}-\left(C_{0,1}^{1,1}\right)^{2}+C_{0,1}^{0,2} C_{0,1}^{2,0}-C_{1,0}^{1,1} C_{0,1}^{2,0}+C_{0,1}^{1,1} C_{1,0}^{2,0}, \\
\delta_{0,0}^{1,1} & =C_{0,0}^{1,1}+C_{0,1}^{1,1} C_{1,0}^{1,1}-C_{1,0}^{0,2} C_{0,1}^{2,0}, \\
\delta_{0,0}^{0,2} & =C_{0,0}^{0,2}-C_{1,0}^{0,2} C_{0,1}^{1,1}+C_{0,1}^{0,2} C_{1,0}^{1,1}-\left(C_{1,0}^{1,1}\right)^{2}+C_{1,0}^{0,2} C_{1,0}^{2,0}, \\
\delta_{h k}^{r_{j}, s_{j}} & =0, \text { otherwise. }
\end{aligned}
$$

We now express the $\rho_{h k}^{q}$, listed in (43), in terms of the $\delta_{h k}^{r_{j}, s_{j}}$, using the idea in the proof of Theorem 6.1.1. In light of Remark 6.2.1 and (54), we see that (52) yields

$$
\left(G_{1}-\mathcal{D}_{1}, G_{2}-\mathcal{D}_{2}, G_{3}-\mathcal{D}_{3}\right)=\left(-\delta_{0,0}^{2,0},-\delta_{0,0}^{1,1},-\delta_{0,0}^{0,2}\right) .
$$

Computing the dot products of the $\mu$-pseudosyzygies (42) with the the preceding vector, as in (51), we obtain

$$
\begin{aligned}
\rho_{0,0}^{1}+\rho_{1,0}^{1} x+\rho_{0,1}^{1} y= & \left(-y+C_{1,0}^{1,1}, x-C_{1,0}^{2,0}+C_{0,1}^{1,1},-C_{0,1}^{2,0}\right) \cdot \\
& \left(-\delta_{0,0}^{2,0},-\delta_{0,0}^{1,1},-\delta_{0,0}^{0,2}\right) \\
= & \left(-C_{1,0}^{1,1} \delta_{0,0}^{2,0}+\left(C_{1,0}^{2,0}-C_{0,1}^{1,1}\right) \delta_{0,0}^{1,1}+C_{0,1}^{2,0} \delta_{0,0}^{0,2}\right)- \\
& \delta_{0,0}^{1,1} x+\delta_{0,0}^{2,0} y, \\
\rho_{0,0}^{2}+\rho_{1,0}^{2} x+\rho_{0,1}^{2} y= & \left(C_{1,0}^{0,2},-y-C_{1,0}^{1,1}+C_{0,1}^{0,2}, x-C_{0,1}^{1,1}\right) \cdot \\
& \left(-\delta_{0,0}^{2,0},-\delta_{0,0}^{1,1},-\delta_{0,0}^{0,2}\right) \\
= & \left(-C_{1,0}^{0,2} \delta_{0,0}^{2,0}+\left(C_{1,0}^{1,1}-C_{0,1}^{0,2}\right) \delta_{0,0}^{1,1}+C_{0,1}^{1,1} \delta_{0,0}^{0,2}\right)- \\
& \delta_{0,0}^{0,2} x+\delta_{0,0}^{1,1} y,
\end{aligned}
$$

from which we deduce

$$
\begin{aligned}
& \rho_{1,0}^{1}=-\delta_{0,0}^{1,1}, \\
& \rho_{0,1}^{1}=\delta_{0,0}^{2,0}, \\
& \rho_{1,0}^{2}=-\delta_{0,0}^{0,2}, \\
& \rho_{0,0}^{1}=-C_{1,0}^{1,1} \delta_{0,0}^{2,0}+\left(C_{1,0}^{2,0}-C_{0,1}^{1,1}\right) \delta_{0,0}^{1,1}+C_{0,1}^{2,0} \delta_{0,0}^{0,2}, \\
& \rho_{0,0}^{2}=-C_{1,0}^{0,2} \delta_{0,0}^{2,0}+\left(C_{1,0}^{1,1}-C_{0,1}^{0,2}\right) \delta_{0,0}^{1,1}+C_{0,1}^{1,1} \delta_{0,0}^{0,2}, \\
& \rho_{0,1}^{2}=\delta_{0,0}^{1,1} .
\end{aligned}
$$

Note that the $\rho_{h k}^{j}$ in the first three of these relations are the elements of the generating set (44) of the ideal $\mathfrak{R}_{\mu}$; one may check that these equations are consistent with the values given in (43) and (54). By replacing the $\delta$ 's in the 
last three relations with their $\rho$-equivalents from the first three relations, we derive the relations (45), as we promised to do.

\section{Smaller generating sets for $\mathcal{O}_{U_{\mu}}$ and affine cell criteria.}

In this, the final section of the paper, we use Theorem 6.1.1 to identify a subset of $\mathfrak{c}_{\mu}$ that generates $\mathcal{O}_{U_{\mu}}$ as a k-algebra, as promised at the end of Section 2; we thereby obtain a smaller explicit presentation of $\mathcal{O}_{U_{\mu}}$ as a quotient of a polynomial ring than those obtained in Sections 5 and 6 . We then derive sufficient conditions on $\mu$ for $U_{\mu}$ to be a $2 n$-dimensional affine cell in $\mathbf{H}^{n}$; more precisely, for the map (15)

$$
\varepsilon_{\mu}: U_{\mu} \rightarrow \operatorname{Spec}\left(\mathrm{k}\left[\mathrm{p}_{\mu}\right]\right)=\mathrm{A}_{\mathrm{k}}^{2 n}
$$

to be an isomorphism.

7.1. A "smaller" set of generators for $\mathcal{O}_{U_{\mu}}$. Recall that $\mathrm{p}_{\mu}$ denotes the $2 n$-member subset (14) of $\mathfrak{c}_{\mu}$ that gives local parameters at the monomial ideal $I_{\mu} \in U_{\mu}$ (8), and is accordingly a k-algebraically independent set. We define

$$
\mathrm{P}_{\mu}=\left\{C_{h k}^{r_{j}, s_{j}} \in \mathfrak{C}_{\mu} \mid c_{h k}^{r_{j}, s_{j}} \in \mathrm{p}_{\mu}\right\},
$$

and observe that the map

$$
\left.u_{\mu}^{*}\right|_{k\left[\mathrm{P}_{\mu}\right]}: \mathrm{k}\left[\mathrm{P}_{\mu}\right] \rightarrow \mathcal{O}_{U_{\mu}}, \quad C_{h k}^{r_{j}, s_{j}} \mapsto c_{h k}^{r_{j}, s_{j}},
$$

the restriction of the surjection (36), is injective.

Recall further that $\mathbf{M}_{\mu}$ denotes the matrix whose rows are the $\mu$-pseudosyzygies $\mathrm{H}_{j}$ of $\left(G_{k}\right)$ discussed in Section 5.1. We define

$$
\begin{aligned}
\operatorname{Ex}_{\mu} & =\left\{C_{h k}^{r_{j}, s_{j}} \in \mathfrak{C}_{\mu} \mid C_{h k}^{r_{j}, s_{j}} \text { appears in } \mathbf{M}_{\mu}\right\} ; \\
\operatorname{ex}_{\mu} & =\left\{c_{h k}^{r_{j}, s_{j}} \mid C_{h k}^{r_{j}, s_{j}} \in \mathrm{Ex}_{\mu}\right\} \subseteq \mathfrak{c}_{\mu} ;
\end{aligned}
$$

the notation recalls that these are the sets of exposed coefficients in the language of Section 4.1. We shall soon show that $\operatorname{ex}_{\mu}$ generates $\mathcal{O}_{U_{\mu}}$ as a k-algebra; we first pause to establish:

Lemma 7.1.1. $\mathrm{P}_{\mu} \subseteq \mathrm{Ex}_{\mu}$; whence, $\mathrm{p}_{\mu} \subseteq \mathrm{ex}_{\mu}$.

Proof. Let $C_{h k}^{r_{j}, s_{j}} \in \mathrm{P}_{\mu}$. Then, by definition of $\mathrm{p}_{\mu}$ (see Section 2.4), one of the following conditions holds:

- $x^{r_{j}} y^{s_{j}}$ is a top leading monomial and $x^{h} y^{k}$ is the rightmost partition monomial in row $h$ (in the diagram of $\mu$ ), with $s_{j} \leq k$, or

- $x^{r_{j}} y^{s_{j}}$ is a side leading monomial and $x^{h} y^{k}$ is the topmost partition monomial in column $k$, with $s_{j}>k$.

In the first case, $C_{h k}^{r_{j}, s_{j}}$ will appear in the $\mu$-pseudosyzygy generated by Algorithm 4.2.1 when $G_{j}$ is multiplied by $-y$; that is, $\mathrm{H}_{j}$; in the second case, 
$C_{h k}^{r_{j}, s_{j}}$ will appear in the $\mu$-pseudosyzygy generated when $G_{j}$ is multiplied by $x$; that is, $\mathbf{H}_{j-1}$. In either case, $C_{h k}^{r_{j}, s_{j}}$ appears in $\mathbf{M}_{\mu}$ (or is exposed), and is therefore a member of $\mathrm{Ex}_{\mu}$, as desired.

We write

$$
\bar{u}_{\mu}^{*}: \mathrm{k}\left[\mathrm{Ex}_{\mu}\right] \rightarrow \mathcal{O}_{U_{\mu}}, \quad C_{h k}^{r_{j}, s_{j}} \mapsto c_{h k}^{r_{j}, s_{j}}
$$

for the restriction of the map (36) to $\mathrm{k}\left[\mathrm{Ex}_{\mu}\right] \subseteq \mathrm{k}\left[\mathfrak{C}_{\mu}\right]$.

Proposition 7.1.2. The map $\bar{u}_{\mu}^{*}$ is surjective, and its kernel is generated by the set $\left\{\delta_{h k}^{r_{j}, s_{j}} \mid C_{h k}^{r_{j}, s_{j}} \in \mathrm{Ex}_{\mu}\right\}$. In particular, the set $\operatorname{ex}_{\mu}$ generates $\mathcal{O}_{U_{\mu}}$ as a k-algebra.

Proof. Recall that $\delta_{h k}^{r_{j}, s_{j}}=C_{h k}^{r_{j}, s_{j}}-D_{h k}^{r_{j}, s_{j}}$ (50), where $D_{h k}^{r_{j}, s_{j}} \in \mathrm{k}\left[\mathrm{Ex} x_{\mu}\right]$ (since a coefficient of a subdeterminant of $\mathbf{M}_{\mu}$ can only involve $C_{h k}^{r_{j}, s_{j}} \in \mathrm{Ex}_{\mu}$ ). We define a surjective map

$$
\begin{aligned}
\tau: \mathrm{k}\left[\mathfrak{C}_{\mu}\right] & \rightarrow \mathrm{k}\left[\mathrm{Ex}_{\mu}\right], \\
C_{h h}^{r_{j}, s_{j}} & \mapsto C_{h k}^{r_{j}, s_{j}} \text { for } C_{h k}^{r_{j}, s_{j}} \in \mathrm{Ex}_{\mu}, \\
C_{h k}^{r_{j}, s_{j}} & \mapsto D_{h k}^{r_{j}, s_{j}} \text { for } C_{h k}^{r_{j}, s_{j}} \notin \mathrm{Ex}_{\mu} .
\end{aligned}
$$

Since $C_{h k}^{r_{j}, s_{j}}$ and $D_{h k}^{r_{j}, s_{j}}$ have the same image under the surjection $u_{\mu}^{*}(49)$, we see that $u_{\mu}^{*}$ factors as

$$
\mathrm{k}\left[\mathfrak{C}_{\mu}\right] \stackrel{\tau}{\rightarrow} \mathrm{k}\left[\mathrm{Ex}_{\mu}\right] \stackrel{\bar{u}_{\mu}^{*}}{\rightarrow} \mathcal{O}_{U_{\mu}}
$$

it follows at once that $\bar{u}_{\mu}^{*}$ is a surjection.

Now let $\kappa$ be an element of $\operatorname{ker}\left(\bar{u}_{\mu}^{*}\right)$, and let $\kappa^{\prime} \in \mathrm{k}\left[\mathfrak{C}_{\mu}\right]$ be any preimage of $\kappa$. Since $\kappa^{\prime} \in \operatorname{ker}\left(u_{\mu}^{*}\right)$, Theorem 6.1 .1 allows us to write

$$
\kappa^{\prime}=\sum f_{h k}^{r_{j}, s_{j}} \cdot \delta_{h k}^{r_{j}, s_{j}}
$$

where the coefficients $f_{h k}^{r_{j}, s_{j}} \in \mathrm{k}\left[\mathfrak{C}_{\mu}\right]$. Since the $\delta_{h k}^{r_{j}, s_{j}}$ corresponding to $C_{h k}^{r_{j}, s_{j}}$ $\notin \mathrm{Ex}_{\mu}$ map to 0 under $\tau$, we may apply $\tau$ to both sides of (57) to obtain a representation of $\kappa$ as a $\mathrm{k}\left[\mathrm{Ex}_{\mu}\right]$-linear combination of the $\delta_{h k}^{r_{j}, s_{j}}$ corresponding to $C_{h k}^{r_{j}, s_{j}} \in \mathrm{Ex}_{\mu}$; this completes the proof of the proposition.

We indicated at the end of Section 2 that $\mathrm{ex}_{\mu}$ is typically a much smaller set of k-algebra generators of $\mathcal{O}_{U_{\mu}}$ than is $\mathfrak{c}_{\mu}$ (but not a minimal generating set, in general). To make this precise, we count the number of elements of $\mathrm{ex}_{\mu}$, using the notation (16): Each of the $p_{1}$ top monomials, when multiplied by $-y$, contributes $\ell$ exposed coefficients to $\mathrm{ex}_{\mu}$; likewise, each of the $\ell$ side monomials, when multiplied by $x$, contributes $p_{1}$ exposed coefficients. However, there are $d_{\mu}-1$ monomials $x^{r} y^{s}$ that are both top and side monomials, and each of these exposes twice the $d_{\mu}$ "northeast corner" coefficients $C_{h k}^{r s}$, where $x^{h} y^{k}$ is both the highest member of its column and the rightmost 
member of its row (see Figure 4). Therefore, the cardinality of $\mathrm{ex}_{\mu}$ is given by

$$
\left|\operatorname{ex}_{\mu}\right|=2\left(p_{1} \cdot \ell\right)-d_{\mu}\left(d_{\mu}-1\right) .
$$

For the example $\mu=(5,3,2,2)$ shown in Figure 4 , we have that $\left|\mathfrak{c}_{\mu}\right|=$ $\Lambda_{\mu} \cdot n=7 \cdot 12=84$, by $(20)$, whereas $\left|\operatorname{ex}_{\mu}\right|=2(5 \cdot 4)-3 \cdot 2=34$.

7.2. Sufficient conditions for $U_{\mu}$ to be an affine cell. We seek conditions under which the map (15)

$$
\varepsilon_{\mu}: U_{\mu} \rightarrow \operatorname{Spec}\left(\mathrm{k}\left[\mathrm{p}_{\mu}\right]\right)=\mathrm{A}_{\mathrm{k}}^{2 n}
$$

is an isomorphism, or, equivalently, conditions under which the inclusion $\mathrm{k}\left[\mathrm{p}_{\mu}\right] \subseteq \mathcal{O}_{U_{\mu}}$ is an equality. Since $\mathcal{O}_{U_{\mu}}$ is generated as a k-algebra by the set $\mathrm{ex}_{\mu}$ (Proposition 7.1.2), we obtain the following:

Corollary 7.2.1. If every element of $\mathrm{ex}_{\mu}$ lies in $\mathrm{k}\left[\mathrm{p}_{\mu}\right]$, then the map $\varepsilon_{\mu}$ is an isomorphism; in particular, $U_{\mu}$ is a $2 n$-dimensional affine cell in $\mathbf{H}^{n}$.

A special case of particular interest occurs when $\mathrm{P}_{\mu}=\mathrm{Ex}_{\mu}$ (which implies $\mathrm{p}_{\mu}=\mathrm{ex}_{\mu}$; recall from Lemma 7.1.1 that $\subseteq$ always holds):

Corollary 7.2.2. If $\mathrm{P}_{\mu}=\mathrm{Ex}_{\mu}$, then the map $\varepsilon_{\mu}$ is an isomorphism, and consequently $U_{\mu}$ is an affine cell. Furthermore, the summands $\mathcal{N}_{j}$ (48) of the polynomials $\mathcal{D}_{j}(47), 1 \leq j \leq \Lambda_{\mu}$, all vanish.

Proof. Only the last statement requires proof, to which end we write

$$
\mathcal{N}_{j}=\sum N_{j}^{p, q} x^{p} y^{q}, \quad N_{j}^{p, q} \in \mathrm{k}\left[\mathfrak{C}_{\mu}\right],
$$

and recall that $\widetilde{u}_{\mu}^{*}\left(\mathcal{N}_{j}\right)=0$, where $\widetilde{u}_{\mu}^{*}$ is the map (37) given by applying $u_{\mu}^{*}$ to each coefficient $N_{j}^{p, q}$. We therefore have that $u_{\mu}^{*}\left(N_{j}^{p, q}\right)=0$, and, by hypothesis, $N_{j}^{p, q} \in \mathrm{k}\left[\mathrm{Ex}_{\mu}\right]=\mathrm{k}\left[\mathrm{P}_{\mu}\right]$. But we noted earlier that the map $\left.u_{\mu}^{*}\right|_{\mathrm{k}\left[\mathrm{P}_{\mu}\right]}(55)$ is injective; whence, all the $N_{j}^{p, q}$ are 0 , as desired.

The case $\mu=(2,1)$, studied in Sections 5.2 and 6.2, provides an example of Corollary 7.2.2. One sees by inspection of $(42)$ that $\mathrm{Ex}_{(2,1)}=\mathrm{P}_{(2,1)}$, and we noted both that $U_{2,1}$ is an affine cell (46) and that the polynomials $\mathcal{D}_{j}$, $j=1,2,3$, all have vanishing summands $\mathcal{N}_{j}$ (Remark 6.2.1). We generalize this example in the following section.

7.3. Necessary and sufficient conditions on $\mu$ for $\mathrm{P}_{\mu}=\mathrm{Ex}_{\mu}$. Recall that we write a partition $\mu=\left(p_{1}, p_{2}, \ldots, p_{\ell}\right)$ of $n$ with parts in decreasing order, and that we use the notations (16); in particular, $\mu(i)$ denotes the number of occurrences of the integer $i$ in $\mu$. 
Proposition 7.3.1. Let $\mu$ be a partition of $n$. In order that $\mathrm{P}_{\mu}=\mathrm{Ex}_{\mu}$, it is necessary and sufficient that

$$
\begin{aligned}
p_{\mu\left(p_{1}\right)+1} & =p_{1}-1, \\
p_{\mu\left(p_{1}\right)+2} & =p_{1}-2, \\
& \vdots \\
p_{\ell}=p_{\mu\left(p_{1}\right)+\left(\ell-\mu\left(p_{1}\right)\right)} & =p_{1}-\left(\ell-\mu\left(p_{1}\right)\right) ;
\end{aligned}
$$

that is, the diagram of $\mu$ has the "sawtooth" form shown in Figure 6, with every step width except possibly the topmost, and every step height except possibly the rightmost, being of size 1.

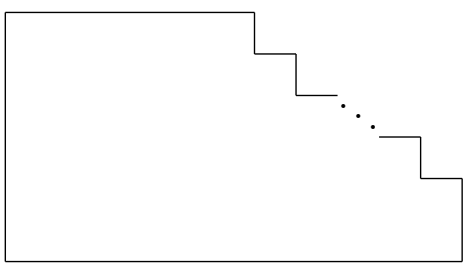

Figure 6. Diagram of $\mu$ satisfying the hypotheses of Proposition 7.3.1.

Proof. We first prove the sufficiency. Suppose that $\mu$ has the indicated form, and consider the top leading monomial $m=x^{r_{j}} y^{s_{j}}$. The monomial $m$ either lies above the topmost horizontal edge of the diagram or in one of the sawtooth "notches." In the former case, each term $C_{h k}^{r_{j}, s_{j}} x^{h} y^{k}$ that is exposed when the polynomial $G_{j}$ is multiplied by $-y$ is positioned at the rightmost end of row $h$ of the diagram, with $s_{j} \leq k$; therefore, by (56), $C_{h k}^{r_{j}, s_{j}} \in \mathrm{P}_{\mu}$. In the latter case, the exposed terms in $-y \cdot G_{j}$ are either of the form just described or of the form $C_{h k}^{r_{j}, s_{j}} x^{h} y^{k}$, with $x^{h} y^{k}$ positioned at the top of column $k$, and $s_{j}>k$; since $m$ is in this case both a top and a side monomial, we conclude that all the exposed $C_{h k}^{r_{j}, s_{j}} \in \mathrm{p}_{\mu}$. Similarly, one checks that if $m$ is a side monomial, then all the exposed coefficients in $x \cdot G_{j}$ lie in $\mathrm{P}_{\mu}$; whence, $\mathrm{Ex}_{\mu} \subseteq \mathrm{P}_{\mu}$, which yields $\mathrm{Ex}_{\mu}=\mathrm{P}_{\mu}$.

It remains to prove the necessity. Arguing by contradiction, we suppose that $\mathrm{Ex}_{\mu}=\mathrm{P}_{\mu}$, but that the diagram of $\mu$ does not have the "sawtooth" form. Suppose that the intermediate horizontal step at height $h$ has width two or greater, that is, the diagram of $\mu$ at height $h$ is as in Figure 7.

$$
\begin{array}{|cccc}
\mathbf{x}^{\mathbf{h}+\mathbf{1}} \mathbf{y}^{\mathbf{s}} & \ldots & \mathbf{x}^{\mathbf{h}+\mathbf{1}} \mathbf{y}^{\mathbf{k}-\mathbf{1}} & \mathbf{x}^{\mathbf{h}+\mathbf{1}} \mathbf{y}^{\mathbf{k}} \\
x^{h} y^{s} & \cdots & x^{h} y^{k-1} & x^{h} y^{k}
\end{array}
$$

Figure 7. A horizontal step of size $>1$. 


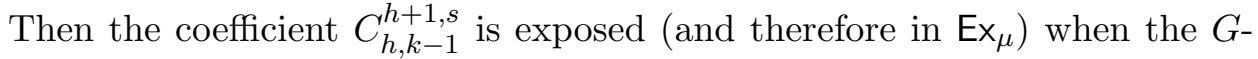
polynomial with leading monomial $x^{h+1} y^{s}$ is multiplied by $x$, but according to (56), this coefficient is not in $\mathrm{P}_{\mu}$, contradicting $\mathrm{Ex}_{\mu} \subseteq \mathrm{P}_{\mu}$. A similar contradiction is obtained if an intermediate vertical step has height two or greater.

Combining the proposition and Corollary 7.2.2, we obtain:

Corollary 7.3.2. If $\mu$ is a partition of $n$ satisfying the hypothesis of Proposition 7.3.1, then the map $\varepsilon_{\mu}$ (15) is an isomorphism; consequently, $U_{\mu}$ is an affine cell. Furthermore, the summands $\mathcal{N}_{j}$ (48) of the polynomials $\mathcal{D}_{j}$ (47), $1 \leq j \leq \Lambda_{\mu}$, all vanish.

\section{Remarks 7.3.3.}

1) Haiman discusses the case $\mu=(1,1, \ldots, 1)$ in detail, from another direction, in [7, p. 214]; his discussion implies that $U_{\mu}$ is an affine cell, with $\mathcal{O}_{U_{\mu}}=\mathrm{k}\left[\mathrm{p}_{\mu}\right]$. Since the diagram of $\mu$ consists of a single column of width 1, with no "sawteeth," it satisfies the hypothesis of Proposition 7.3.1, and therefore Haiman's results also follow from Corollary 7.3.2.

2) In case $\mu=(r, r-1, \ldots, 1)$, a partition of $n=r(r+1) / 2$, it is clear that the hypothesis of Proposition 7.3.1 is satisfied; whence, $U_{\mu}$ is an affine cell (this is a direct generalization of the case $\mu=(2,1)$ considered earlier). The affine cellularity of $U_{\mu}$ can be obtained in another way by suitably modifying [8, Corollary 4.11 and Example 4.13]. (Similarly, [8, Example 4.12] concerns $\mu=(1,1, \ldots, 1)$, and yields yet another proof of the affine cellularity in that case.) Note that the monomial ideal $I_{\mu} \in U_{\mu}$ is the "fat point" ideal $(x, y)^{r}=\left(x^{r}, x^{r-1} y, \ldots, y^{r}\right) \subseteq$ $\mathrm{k}[x, y]$.

7.4. A sufficient condition on $\mu$ for $\mathrm{ex}_{\mu} \subseteq \mathrm{k}\left[\mathrm{p}_{\mu}\right]$. We now further generalize the criterion for affine cellularity proved in Section 7.3 by exploiting Corollary 7.2.1, the hypothesis of which is that $\mathrm{ex}_{\mu} \subseteq \mathrm{k}\left[\mathrm{p}_{\mu}\right]$. Since it is inconvenient for our current purpose to use the indexing of the leading monomials by $j, 1 \leq j \leq \Lambda_{\mu}$, we will drop this index from the notations $x^{r_{j}} y^{s_{j}}, C_{h k}^{r_{j}, s_{j}}$, etc., and we will denote the polynomials $G_{j}, g_{j}$ by $G_{(r, s)}, g_{(r, s)}$, respectively. Recall from (16) that we write $d_{\mu}$ for the number of distinct parts of the partition $\mu$, and $\mu(i)$ for the number of times the integer $i$ occurs in $\mu$.

Theorem 7.4.1. Let $\mu$ be a partition of $n$ with distinct parts $p_{1}=s_{1}$, $s_{2}, \ldots, s_{d_{\mu}}=p_{\ell}$ such that

$$
\begin{gathered}
s_{2}=s_{1}-1, s_{3}=s_{2}-1, \ldots, s_{d_{\mu}}=s_{d_{\mu}-1}-1, \text { and } \\
\mu\left(s_{d_{\mu}}\right) \leq \mu\left(s_{d_{\mu}-1}\right) \leq \cdots \leq \mu\left(s_{2}\right)
\end{gathered}
$$


that is, such that the diagram of $\mu$ has the shape shown in Figure 8: Every step except possibly for the first is of width 1 , and the step heights $\mu\left(s_{t}\right)$ are nondecreasing as one moves to the right, except for the possibility that at the last stage $\mu\left(s_{2}\right)>\mu\left(s_{1}\right)$. Then $\mathrm{ex}_{\mu} \subseteq \mathrm{k}\left[\mathrm{p}_{\mu}\right]$.

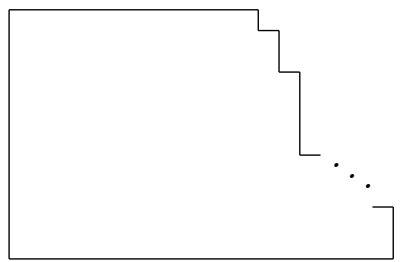

Figure 8. Diagram of $\mu$ satisfying the hypothesis of Theorem 7.4.1.

7.4.1. Start of the proof of Theorem 7.4.1. Recall that every leading monomial $x^{r} y^{s}$ has either one or two opportunities to contribute coefficient functions to $\mathrm{ex}_{\mu}$ as the syzygies of the $g_{\left(r^{\prime}, s^{\prime}\right)}$ are formed: Top leading monomials contribute the exposed coefficients in $-y \cdot g_{(r, s)}$, side leading monomials contribute the exposed coefficients in $x \cdot g_{(r, s)}$, and some leading monomials contribute in both ways. We say that a coefficient function $c_{h k}^{r, s}$ is covered provided that it is a member of $\mathrm{k}\left[\mathrm{p}_{\mu}\right]$. Accordingly, we say that a leading monomial is covered for $\mathbf{y}$ (resp. $\mathbf{x}$ ) if its contributions to $\mathrm{ex}_{\mu}$ via $-y \cdot g_{(r, s)}$ (resp. $x \cdot g_{(r, s)}$ ) are all covered. To prove the theorem, we must prove that every top leading monomial is covered for $y$, and every side leading monomial is covered for $x$.

To do this, it is convenient to group the leading and partition monomials by columns; that is, by $y$-degree $k, 0 \leq k \leq p_{1}=s_{1}$. We use the following notation: In the column of $y$-degree $k$, we write $x^{r_{k}} y^{k}$ for the leading monomial of least $x$-degree in the column; if $k<p_{1}$, this is the unique top leading monomial in the column (see Figure 9). One checks easily that

$$
r_{k}=\mu(k+1)+r_{(k+1)}=\mu(k+1)+\mu(k+2)+\cdots+\mu\left(p_{1}\right),
$$

and that the number $L(k)$ of leading monomials in column $k$ is given by

$$
L(k)=\left\{\begin{array}{l}
1, \text { for } 0 \leq k \leq p_{\ell}-1 \\
\mu(k) \geq 1, \text { for } p_{\ell} \leq k \leq p_{1} .
\end{array}\right.
$$

To help keep track of the coefficient functions $c_{h k}^{r, s}$ that we have shown are covered, we introduce, for each pair of column indices $(s, k)$ with $0 \leq s \leq$ 


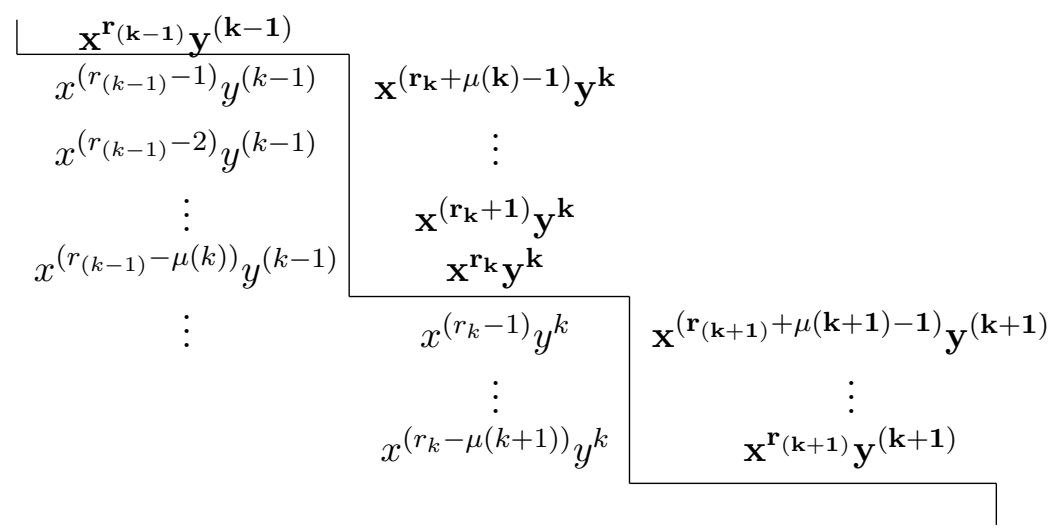

Figure 9. The (intermediate) column of $y$-degree $k$ and its neighbors in the diagram of $\mu$ (leading monomials shown in bold).

$p_{1}, 0 \leq k \leq p_{1}-1$, the matrix

$$
\mathcal{C}(s, k)=\left[\begin{array}{ccc}
c_{r_{k}-1, k}^{r_{s}+L(s)-1, s} & c_{r_{k}-2, k}^{r_{s}+L(s)-1, s} & \ldots \\
c_{r_{k}-1, k}^{r_{s}+L(s)-2, s} & c_{r_{k}-2, k}^{r_{s}+L(s)-2, s} & \ldots \\
\vdots & \vdots & \\
c_{r_{k}-1, k}^{r_{s}, s} & c_{r_{k}-2, k}^{r_{s}, s} & \ldots
\end{array}\right]
$$

that is, the matrix with $L(s)$ rows, arbitrarily many columns, and entries given by

$$
\mathcal{C}(s, k)_{i, j}=c_{r_{k}-j, k}^{r_{s}+L(s)-i, s}
$$

where we define the entries to be 0 whenever $r_{k}-j<0$ (in other words, the columns to the right of the $r_{k}$-th column are all 0 ).

We now catalogue the current state of our knowledge and summarize what remains to be proved in terms of the matrices $\mathcal{C}(s, k)$ (initially, we know that the coefficient functions $c_{h k}^{r, s} \in \mathrm{p}_{\mu}$ are trivially covered). We first consider a side leading monomial $x^{r} y^{s}$ (the hypothesis of Theorem 7.4.1 implies that $x^{r} y^{s}$ is a side monomial $\left.\Leftrightarrow s \geq p_{\ell}\right)$. Note that the exposed coefficients in $x \cdot g_{(r, s)}$ are the $c_{r_{k}-1, k}^{r, s}, 0 \leq k<p_{1}$. By $(56), c_{r_{k}-1, k}^{r, s} \in \mathrm{p}_{\mu}$ provided that $s$ $>k$. Expressed in terms of the matrices $\mathcal{C}(s, k)$, the last conclusion reads as follows: Every entry in the first column of $\mathcal{C}(s, k)$ is covered provided that $p_{\ell}$ $\leq s \leq p_{1}, 0 \leq k<s$. To prove that every side leading monomial is covered for $x$, it remains to prove that the entries in the first column of $\mathcal{C}(s, k)$ are all covered for $p_{\ell} \leq s \leq k \leq p_{1}-1$.

We next consider a top leading monomial $x^{r} y^{s}=x^{r_{s}} y^{s}, 0 \leq \mathrm{s} \leq p_{1}-1$. Note that the exposed coefficients in $-y \cdot g_{\left(r_{s}, s\right)}$ are the $c_{h, k}^{r_{s}, s}$ with $p_{\ell}-1 \leq k$ 
$\leq p_{1}-1, r_{k}-\mu(k+1) \leq h \leq r_{k}-1$ (since these correspond to the partition monomials in column $k$ that are rightmost in their respective rows $h$, as illustrated in Figure 9). By (56), these coefficients lie in $\mathrm{p}_{\mu}$ provided that $s$ $\leq k$; in other words, we have that the first $\mu(k+1)$ entries in the bottom row of the matrix $\mathcal{C}(s, k)$ are covered for $s, k$ in the given ranges and $s \leq$ $k$. (Note that when $k=p_{1}-1$, every entry in the bottom row of $\mathcal{C}(s, k)$ is covered, since those of index $j>\mu(k+1)\left(=\mu\left(p_{1}\right)=r_{p_{1}-1}=r_{k}\right)$ are equal to 0 by definition.) To prove that every top leading monomial is covered for $y$, it remains to prove that the first $\mu(k+1)$ entries in the bottom row of the matrix $\mathcal{C}(s, k)$ are covered for $p_{\ell}-1 \leq k<s \leq p_{1}-1$.

In summary, we know that:

- If $p_{\ell} \leq s \leq p_{1}, 0 \leq k<s$, then the $c$ 's in the first column of $\mathcal{C}(s, k)$ are all covered, and

- if $0 \leq \mathrm{s} \leq p_{1}-1, p_{\ell}-1 \leq k \leq p_{1}-1$, and $s<=k$ then the first $\mu(k+1) c$ 's on the bottom row of $\mathcal{C}(s, k)$ are all covered, and all the entries on the bottom row of $\mathcal{C}(s, k)$ are covered if $k=p_{1}-1$,

and it remains to prove that:

- If $p_{\ell} \leq s<p_{1}, s \leq k<p_{1}$, then the $c$ 's in the first column of $\mathcal{C}(s, k)$ are all covered, and

- if $p_{\ell}-1 \leq k<s \leq p_{1}-1$, then the first $\mu(k+1)$ c's on the bottom row of $\mathcal{C}(s, k)$ are all covered.

We can dispose of one case right away: If the partition $\mu$ contains only one distinct part (i.e., $p_{1}=p_{\ell}$ ), then the hypothesis of Theorem 7.4.1 is clearly satisfied and (60) is vacuously true, so the desired conclusion ( $\mathrm{ex}_{\mu}$ $\subseteq \mathrm{k}\left[\mathrm{p}_{\mu}\right]$ ) follows. (Note that Proposition 7.3.1 yields this conclusion in the form $\mathrm{p}_{\mu}=\mathrm{ex}_{\mu}$.) Therefore, we may henceforth assume that $\mu$ has at least two distinct parts.

7.4.2. Reduction of the proof of Theorem 7.4.1 to a lemma. To prove (60), we will in fact prove more: In each case the goal is to establish that the first column (resp. initial segment of the bottom row) of a matrix $\mathcal{C}(s, k)$ is covered. We will do this by showing that the entire lower-left triangular portion of the matrix having the first column (resp. initial segment of the bottom row) in question at its vertical (resp. horizontal) leg is covered; see Figure 10.

More precisely, Theorem 7.4.1 is an immediate consequence of the following:

Lemma 7.4.2. Let the partition $\mu$ satisfy the hypothesis of Theorem 7.4.1 and have at least two distinct parts. Then, for each $s, p_{\ell} \leq s \leq p_{1}-1$, we have that: 


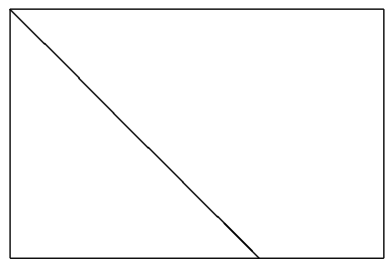

Figure 10. Lower-left triangular portion of the matrix $\mathcal{C}(s, k)$ having the first column as its vertical leg.

1) If $s \leq k \leq p_{1}-1$, the entries $\mathcal{C}(s, k)_{(i, j)}$ of the matrix $\mathcal{C}(s, k)$ lying in the lower-left triangular region defined by $i-j \geq 0$ (which includes the entire first column) are all covered.

2) If $s-1 \leq k<s^{\prime} \leq p_{1}-1$, the entries $\mathcal{C}\left(s^{\prime}, k\right)_{(i, j)}$ of the matrix $\mathcal{C}\left(s^{\prime}, k\right)$ lying in the lower-left triangular region defined by $i-j \geq \mu\left(s^{\prime}\right)-\mu(k+1)$ (which includes the first $\mu(k+1)$ entries on the bottom row) are all covered.

The proof of the lemma proceeds by descending induction on $s$ in the nonempty range $p_{\ell} \leq s \leq p_{1}-1$. A key ingredient is the identity (11), which when recast in terms of the matrices $\mathcal{C}(s, k)$ reads as follows (for $2 \leq$ $i \leq \mu(s))$ :

$$
\mathcal{C}(s, k)_{(i-1, j)}=\mathcal{C}(s, k)_{(i, j+1)}+\sum_{k^{\prime}=0}^{p_{1}-1} \mathcal{C}\left(s, k^{\prime}\right)_{(i, 1)} \cdot \mathcal{C}\left(k^{\prime}, k\right)_{\left(L\left(k^{\prime}\right), j\right)}
$$

7.4.3. Proof of Lemma 7.4.2. Setting aside the base case for the moment, we make the inductive hypothesis that for some $s, p_{\ell} \leq s \leq p_{1}-1$, we have shown that the statements of the lemma hold for all integers in the interval $\left[s+1, p_{1}-1\right]$. We proceed to show that the statements of the lemma hold for $s$.

We begin with the proof of the first statement: We must show that in each of the matrices $\mathcal{C}(s, k)$ with $s \leq k \leq p_{1}-1$, the lower-left triangular region having the entire first column as vertical leg is covered. At the outset, we know by (59) that an initial segment of the bottom row of each $\mathcal{C}(s, k)$ is covered; in particular, the element $\mathcal{C}(s, k)_{(L(s), 1)}$ in the lower-left corner is covered. If $L(s)=\mu(s)=1$, we are done, so we assume that $\mu(s)>1$ and that for each $k^{\prime}$ in the interval $\left[s, p_{1}-1\right]$, the lower-left triangular region of $\mathcal{C}\left(s, k^{\prime}\right)$ defined by $i-j \geq \mu(s)-t>0$ is covered. Choose a particular $k$ in $\left[s, p_{1}-1\right]$ and entry $(i, j)$ of $\mathcal{C}(s, k)$ such that $i-j=\mu(s)-t$, and consider 
the identity (61) specialized to this situation:

$$
\begin{aligned}
\mathcal{C}(s, k)_{(i-1, j)}= & \mathcal{C}(s, k)_{(i, j+1)}+\sum_{k^{*}=0}^{s-1} \mathcal{C}\left(s, k^{*}\right)_{(i, 1)} \cdot \mathcal{C}\left(k^{*}, k\right)_{\left(L\left(k^{*}\right), j\right)} \\
& +\sum_{k^{\prime}=s}^{p_{1}-1} \mathcal{C}\left(s, k^{\prime}\right)_{(i, 1)} \cdot \mathcal{C}\left(k^{\prime}, k\right)_{\left(L\left(k^{\prime}\right), j\right)} .
\end{aligned}
$$

Note that $\mathcal{C}(s, k)_{(i-1, j)}$ lies immediately "northwest" of $\mathcal{C}(s, k)_{(i, j+1)}$ along the diagonal lying immediately above the lower-left triangular region that we have inductively assumed is covered. We claim that the remaining matrix entries on the right-hand side of (62) are all covered:

- $\mathcal{C}\left(s, k^{*}\right)_{(i, 1)}$ is covered by $(59)$, since it is in the first column and $k^{*}<$ $s$

- $\mathcal{C}\left(k^{*}, k\right)_{\left(L\left(k^{*}\right), j\right)}$ is covered by $(59)$, since it lies on the bottom row, $k^{*}$ $<s \leq k$, and for $k<p_{1}-1, j<\mu(s) \leq \mu(k+1)$ (the penultimate inequality holds since $i \leq L(s)=\mu(s)$ and $i-j=\mu(s)-t>0$, and the last reflects the nondecreasing stepsize hypothesis on the partition $\mu$, which is here invoked for the first time in the proof);

- $\mathcal{C}\left(s, k^{\prime}\right)_{(i, 1)}$ is covered because it lies in the lower-left triangular region that we are inductively assuming is covered, since $i-1 \geq i-j=$ $\mu(s)-t$

- $\mathcal{C}\left(k^{\prime}, k\right)_{\left(L\left(k^{\prime}\right), j\right)}$ is covered for one of two reasons: If $k^{\prime} \leq k$, this matrix entry is covered by (59), since it lies on the bottom row and, for $k<$ $p_{1}-1, j<\mu(s) \leq \mu(k+1)$ as in the second bullet. If $k^{\prime}>k$, this matrix entry is covered because of the inductive hypothesis concerning $s$ : Such a $k^{\prime}$ is in the interval $\left[s+1, p_{1}-1\right]$ and $k \geq s$, so the second statement of the lemma applies and tells us that the lower-left triangular region of $\mathcal{C}\left(k^{\prime}, k\right)$ defined by $i-j \geq \mu\left(k^{\prime}\right)-\mu(k+1)$ is covered. Our entry lies in this region since $L\left(k^{\prime}\right)-j=\mu\left(k^{\prime}\right)-j \geq \mu\left(k^{\prime}\right)-\mu(s) \geq \mu\left(k^{\prime}\right)-\mu(k+1)$, where we have again used the inequalities $j<\mu(s) \leq \mu(k+1)$ that follow as before.

It follows that whenever $\mathcal{C}(s, k)_{(i, j+1)}$ is covered, so too is its northwest neighbor $\mathcal{C}(s, k)_{(i-1, j)}$. We claim that the matrix entry lying farthest to the southeast (and therefore in the last row) on the diagonal containing the latter entries is covered: This entry has indices $(\mu(s),(t+1))$, where $i-j=\mu(s)-t>0$. By (59), the first $\mu(k+1)$ entries in the last row of $\mathcal{C}(s, k)$ are covered, and all entries in the last row are covered if $k=p_{1}-1$. If $k<p_{1}-1$, we have $t+1 \leq \mu(s) \leq \mu(k+1)$ (as before); whence, the claim. Therefore, starting at the southeasternmost element and proceeding stepwise to the northwest along our diagonal, we obtain that the lower-left triangular region of $\mathcal{C}(s, k)$ defined by $i-j \geq \mu(s)-(t+1)$ is covered. Since $k$ was chosen arbitrarily in $\left[s, p_{1}-1\right]$, we may conclude by induction on $t$ 
that the lower-left triangular region defined by $i-j \geq 0$ is covered for each of the matrices $\mathcal{C}(s, k)$, as $k$ ranges over the interval $\left[s, p_{1}-1\right]$. This completes the proof (under the inductive hypothesis on $s$ ) that the first statement of the lemma holds for $s$.

We now prove that the second statement of the lemma holds for $s$. In light of the induction hypothesis on $s$, it remains to prove that for every $s^{\prime}$ in the interval $\left[s, p_{1}-1\right]$, the lower-left triangular region of the matrix $\mathcal{C}\left(s^{\prime}, s-1\right)$ defined by $i-j \geq \mu\left(s^{\prime}\right)-\mu(s)$ is covered. By (59), the first column of each $\mathcal{C}\left(s^{\prime}, s-1\right)$ is covered; in particular, the lower left-hand element $\mathcal{C}\left(s^{\prime}, s-1\right)_{\left(L\left(s^{\prime}\right), 1\right)}$ comprising the lower-left triangular region defined by $i-j$ $\geq L\left(s^{\prime}\right)-1=\mu\left(s^{\prime}\right)-1$ is covered. If $\mu(s)=1$, we are done, so suppose that $\mu(s)>1$ and that for all $s^{\prime}$ in $\left[s, p_{1}-1\right]$, the lower-left triangular region of $\mathcal{C}\left(s^{\prime}, s-1\right)$ defined by $i-j \geq \mu\left(s^{\prime}\right)-t>\mu\left(s^{\prime}\right)-\mu(s) \geq 0$ is covered (the last inequality follows from the nondecreasing stepsize hypothesis on $\mu)$. Choose a particular value $\widetilde{s}$ among the $s^{\prime}$ and an entry $(i, j)$ in $\mathcal{C}(\widetilde{s}, s-1)$ such that $i-j=\mu(\widetilde{s})-t$, and consider the identity (61) specialized to this situation:

$$
\begin{aligned}
\mathcal{C}(\widetilde{s}, s-1)_{(i-1, j)}= & \mathcal{C}(\widetilde{s}, s-1)_{(i, j+1)} \\
& +\sum_{k^{*}=0}^{s-1} \mathcal{C}\left(\widetilde{s}, k^{*}\right)_{(i, 1)} \cdot \mathcal{C}\left(k^{*}, s-1\right)_{\left(L\left(k^{*}\right), j\right)} \\
& +\sum_{k^{\prime}=s}^{p_{1}-1} \mathcal{C}\left(\widetilde{s}, k^{\prime}\right)_{(i, 1)} \cdot \mathcal{C}\left(k^{\prime}, s-1\right)_{\left(L\left(k^{\prime}\right), j\right)} .
\end{aligned}
$$

We claim that the matrix entries on the right-hand side of (63), except possibly for the first, are covered:

- $\mathcal{C}\left(\widetilde{s}, k^{*}\right)_{(i, 1)}$ is covered by $(59)$, since it lies in the first column and $k^{*}$ $<\widetilde{s}$

- $\mathcal{C}\left(k^{*}, s-1\right)_{\left(L\left(k^{*}\right), j\right)}$ is covered by $(59)$, since it lies in the last row, $k^{*}$ $\leq s-1$, and $j \leq t<\mu(s)=\mu((s-1)+1)$ (the double inequality holds since $i-j=\mu(\widetilde{s})-t>\mu(\widetilde{s})-\mu(s)$, and $i$, being a row index in $\mathcal{C}(\widetilde{s}, s-1)$, is $\leq L(\widetilde{s})=\mu(\widetilde{s}))$;

- $\mathcal{C}\left(\widetilde{s}, k^{\prime}\right)_{(i, 1)}$ is covered for one of two reasons: If $k^{\prime}<\widetilde{s}$, this entry is covered by (59). If $\widetilde{s} \leq k^{\prime}$, this entry is covered by the first statement of Lemma 7.4.2, already verified by induction to hold for all $s^{\prime}$ in the interval $\left[s, p_{1}-1\right]$;

- $\mathcal{C}\left(k^{\prime}, s-1\right)_{\left(L\left(k^{\prime}\right), j\right)}$ is covered by our induction hypothesis (namely, that the lower-left triangular region of $\mathcal{C}\left(s^{\prime}, s-1\right)$ defined by $i-j \geq \mu\left(s^{\prime}\right)-t$ is covered for all $s^{\prime}$ in the interval $\left.\left[s, p_{1}-1\right]\right)$, since the inequality $j$ $\leq t$ noted in the second bullet implies that $L\left(k^{\prime}\right)-j=\mu\left(k^{\prime}\right)-j \geq$ $\mu\left(k^{\prime}\right)-t$. 
It follows that if $\mathcal{C}(\widetilde{s}, s-1)_{(i-1, j)}$ is covered, then so is $\mathcal{C}(\widetilde{s}, s-1)_{(i, j+1)}$; therefore, beginning with the extreme northwest member of the diagonal containing these entries (which, being in the first column, is covered, as noted earlier), and proceeding stepwise from northwest to southeast, we obtain that the entire diagonal is covered; whence, the larger lower-left triangular region defined by $i-j \geq \mu(\widetilde{s})-(t+1) \geq \mu(\widetilde{s})-\mu(s)$ is covered. Since $\widetilde{s}$ was arbitrarily chosen among the $s^{\prime}$, the last conclusion applies to them all; whence, we may conclude by induction on $t$ that, for all $s^{\prime}$ in the interval $\left[s, p_{1}-1\right]$, the lower-left triangular region of the matrix $\mathcal{C}\left(s^{\prime}, s-1\right)$ defined by $i-j \geq \mu\left(s^{\prime}\right)-\mu(s)$ is covered. This completes the proof (under the inductive hypothesis on $s$ ) that the second statement of Lemma 7.4.2 holds for $s$.

To complete the proof of the lemma, it remains to prove the base case for the descending induction, that is, that the statements of the lemma hold for $s=p_{1}-1$. One sees easily that these statements reduce to the following:

1) The entries in the lower-left triangular region of $\mathcal{C}\left(p_{1}-1, p_{1}-1\right)$ defined by $i-j \geq 0$ are all covered.

2) The entries in the lower-left triangular region of $\mathcal{C}\left(p_{1}-1, p_{1}-2\right)$ defined by $i-j \geq 0$ are all covered.

We leave the proofs as exercises for the reader; simpler versions of the arguments used earlier suffice. This completes the proof of Lemma 7.4.2 and Theorem 7.4.1.

7.5. Further sufficient conditions for $U_{\mu}$ to be an affine cell. The following result is an immediate consequence of Theorem 7.4.1 and Corollary 7.2.1.

Corollary 7.5.1. If $\mu$ is a partition satisfying the hypothesis of Theorem 7.4.1, then the map $\varepsilon_{\mu}(15)$ is an isomorphism; consequently, $U_{\mu}$ is an affine cell.

A simple observation affords a slight generalization: The isomorphism $\tau: \mathrm{A}_{\mathrm{k}}^{2} \rightarrow \mathrm{A}_{\mathrm{k}}^{2}$, defined by

$$
\tau^{*}: \mathrm{k}[x, y] \rightarrow \mathrm{k}[x, y], \quad x \mapsto y, y \mapsto x,
$$

induces (by pullback of subschemes) an isomorphism $\mathbf{H}^{n} \rightarrow \mathbf{H}^{n}$, under which $I \in \mathbf{H}^{n}$ maps to $\tau^{*}(I)$. If $I \in U_{\mu}$, then $\tau^{*}(I) \in U_{\mu^{\prime}}$, where $\mu^{\prime}$ is the conjugate partition to $\mu$, that is, the partition whose diagram is obtained from the diagram of $\mu$ by interchanging rows and columns, so that $(h, k) \in \mu$ if and only if $(k, h) \in \mu^{\prime}$. It follows easily that $\tau$ induces an isomorphism

$$
\begin{gathered}
\tau_{\mu}: U_{\mu} \rightarrow U_{\mu^{\prime}} \text { with comorphism } \\
\tau_{\mu}^{*}: \mathcal{O}_{U_{\mu^{\prime}}} \rightarrow \mathcal{O}_{U_{\mu}} \text { defined by } \hat{c}_{h k}^{p q} \mapsto c_{k h}^{q p}
\end{gathered}
$$


(we write $\hat{c}$ for the $c$ 's in $\mathcal{O}_{U_{\mu^{\prime}}}$ ) and inducing a bijection $\mathrm{p}_{\mu^{\prime}} \rightarrow \mathrm{p}_{\mu}$; whence, we obtain:

Proposition 7.5.2. $U_{\mu}$ is an affine cell if and only if $U_{\mu^{\prime}}$ is an affine cell; moreover, the map $\varepsilon_{\mu^{\prime}}$ is an isomorphism if and only if $\varepsilon_{\mu}$ is an isomorphism.

For example, the partition $\mu=(4,3,3)$ satisfies the hypothesis of Theorem 7.4.1; therefore, both $U_{\mu}$ and $U_{\mu^{\prime}}$ are affine cells, where the conjugate partition $\mu^{\prime}=(3,3,3,1)$. Note that the latter partition does not satisfy the hypothesis of Theorem 7.4.1.

We end this paper with two brief remarks that complement the results obtained here:

\section{Remarks 7.5.3.}

1) By direct computation it can be shown that the map $\varepsilon_{\mu}$ is an isomorphism (and therefore $U_{\mu}$ is an affine cell) in case $\mu=(3,2,1,1)$; however, neither $\mu$ nor its conjugate $\mu^{\prime}=(4,2,1)$ satisfy the hypothesis of Theorem 7.4.1.

2) Haiman observes that $U_{\mu}$ need not be an affine cell for every $\mu[7$, footnote, p. 207].

\section{References}

[1] A. Altman and S. Kleiman, Introduction to Grothendieck duality theory, Lecture Notes in Mathematics, 146, Springer-Verlag, New York, Heidelberg, Berlin, 1970, MR 43 \#224, Zbl 0215.37201.

[2] L. Burch, On ideals of finite homological dimension in local rings, Proceedings of the Cambridge Philosophical Society, 64 (1968), 941-948, MR 37 \#5208, Zbl 0172.32302.

[3] D. Eisenbud, Commutative Algebra with a View Toward Algebraic Geometry, Springer-Verlag, Graduate Texts in Mathematics, 150 (1995), MR 97a:13001, Zbl 0819.13001.

[4] J. Fogarty, Algebraic families on an algebraic surface, Amer. J. Math., 90 (1968), 511-521, MR 38 \#5778, Zbl 0176.18401.

[5] A. Grothendieck, Techniques de construction et théorèmes d'existence en géométrie algébrique, IV: Les schemas de Hilbert, Séminaire Bourbaki, 221, IHP, Paris, 1961, CMP 1611 822, Zbl 0236.14003.

[6] A. Grothendieck and J.A. Dieudonne, Eléments de Géométrie Algébrique, I, SpringerVerlag, New York, Heidelberg, Berlin, 1971, MR 55 \#5621, Zbl 0203.23301.

[7] M. Haiman, T,Q-Catalan numbers and the Hilbert scheme, Discrete Math., 193(1-3) (1998), 201-224, MR 2000k:05264.

[8] M. Huibregtse, Some amplifications and a correction of Iarrobino's construction of local parameters on Hilb $\mathrm{P}_{\mathrm{k}}^{2}$, Rocky Mountain J. Math., 29(4) (1999), 1321-1361, MR 2001c:14008. 
[9] A. Iarrobino, Punctual Hilbert Schemes, Memoirs of the AMS, 10(188), American Mathematical Society, Providence, RI, 1977, MR 58 \#5667, Zbl 0355.14001.

[10] I. Kaplansky, Commutative Rings, Allyn and Bacon, Boston, 1970, MR 40 \#7234, Zbl 0203.34601.

[11] M. Schaps, Deformations of Cohen-Macaulay schemes of codimension 2 and nonsingular deformations of space curves, Amer. J. Math., 99(4) (1977), 669-685, MR 58 \#10918, Zbl 0358.14006.

Received September 6, 2000 and revised July 17, 2001. The author thanks the Department of Mathematics at Texas A\&M University for its hospitality during the writing of the first version of this paper, and Skidmore College for sabbatical support. He also thanks the referee for many helpful suggestions.

Department of Mathematics and Computer Science

Skidmore College

Saratoga Springs, New York 12866

E-mail address: mhuibreg@skidmore.edu 